\title{
Un scrutin pour trois pays : la nouvelle géographie politique italienne suite à l'élection législative du 4 mars 2018
}

\author{
Matteo Cavallaro, Davide Policastro, Guido Salza et Massimo Angelo \\ Zanetti
}

\section{(2) OpenEdition}

Édition électronique

URL : http://journals.openedition.org/echogeo/16294

DOI : $10.4000 /$ echogeo.16294

ISSN : 1963-1197

Éditeur

Pôle de recherche pour l'organisation et la diffusion de l'information géographique (CNRS UMR 8586)

Référence électronique

Matteo Cavallaro, Davide Policastro, Guido Salza et Massimo Angelo Zanetti, « Un scrutin pour trois pays : la nouvelle géographie politique italienne suite à l'élection législative du 4 mars 2018 », EchoGéo [En ligne], Sur le Vif, mis en ligne le 31 décembre 2018, consulté le 28 septembre 2019. URL : http:// journals.openedition.org/echogeo/16294; DOI : 10.4000/echogeo.16294

Ce document a été généré automatiquement le 28 septembre 2019.

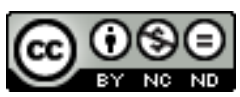

EchoGéo est mis à disposition selon les termes de la licence Creative Commons Attribution - Pas d'Utilisation Commerciale - Pas de Modification 4.0 International 


\title{
Un scrutin pour trois pays : la nouvelle géographie politique italienne suite à l'élection législative du 4 mars 2018
}

\author{
Matteo Cavallaro, Davide Policastro, Guido Salza et Massimo Angelo \\ Zanetti
}

\section{Introduction}

1 Le 4 mars 2018, dans un contexte politique et économique très incertain, les électeurs italiens ont voté pour renouveler le Parlement. L'Italie étant une démocratie parlementaire, ce sont les résultats de l'élection des deux chambres composant le Parlement, la Camera dei Deputati (Chambre des députés) et le Senato (Senat), qui déterminent l'orientation politique de l'exécutif. Le scrutin du 4 mars a confirmé la rupture nette, déjà évidente lors de l'élection législative de 2013, avec le panorama politique italien de l'avant-crise. Son résultat marque la fin de la Seconde République, période de l'histoire politique italienne commencée avec la chute du mur de Berlin et caractérisée par le rôle central de Silvio Berlusconi, leader du centre-droit et de son parti « personnel », Forza Italia (Force Italie - FI).

2 Le système politique apparaît bouleversé par une profonde crise économique. Comme nous pouvons l'observer dans le tableau 1, qui montre les résultats du scrutin ainsi que le groupe d'appartenance au Parlement Européen des différentes listes, le vote italien du 4 mars a été marqué par trois éléments: (i) l'affirmation des forces dites « populistes» (la Lega et le Movimento 5 Stelle, M5S) qui, divisées lors du scrutin, se sont alliées post-élection au Parlement et sont aujourd'hui conjointement au gouvernement; (ii) l'effondrement du centre-gauche, réuni autour du Partito Democratico (PD), qui a atteint son point le plus bas dans l'histoire de l'Italie républicaine et (iii) la lutte interne pour la leadership du centre-droit entre la Lega 
(Ligue) de Matteo Salvini et le parti de Silvio Berlusconi, FI. La lutte a nettement été gagnée par la Ligue di Salvini, qui s'est donné pour projet de sortir de son (auto)confinement traditionnel territorial dans les régions du Nord afin de devenir un parti national ayant désormais remplacé FI à la tête de la coalition de centre-droit.

3 Cet article est consacré à l'analyse du vote du 4 mars, en particulier à la nouvelle géographie politique sortie des urnes. Derrière cette carte inédite du Belpaese, il est possible de lire, en filigrane, le profil social de l'électorat de chaque force : certes, il faut se garder d'un déterminisme électoral mais il demeure possible d'identifier les caractéristiques sociodémographiques des territoires plus favorables à l'une ou à l'autre coalition. Les clivages sociaux (salariés / auto-entrepreneurs, jeunes / vieux, chômeurs / employés, etc.) sur lesquels se joue une élection peuvent être appréciés à travers une dimension spatiale; le contexte dans lequel vous vivez interagit avec le "qui vous êtes» et contribue au choix du vote de chaque électeur. Nous nous demanderons donc quelles sont les spécificités sociales et économiques qui caractérisent les territoires des forces politiques majeures dans cette nouvelle période du système politique italien.

Le travail se divise en quatre parties. Nous commencerons par présenter le contexte du scrutin, notamment en revenant sur l'histoire de la géographie politique italienne, longtemps stable, qui peut se diviser en deux grandes périodes avant la rupture actuelle : la Première et la Deuxième Républiques. Les règles du jeu, c'est-à-dire la nouvelle loi électorale, adoptée en Novembre 2017 et utilisée pour la première fois lors du scrutin du 4 mars, seront ensuite explicitées. La deuxième partie est consacrée à la présentation et à l'analyse des résultats, (taux de participation, distribution et variation sur le territoire du consensus des trois coalitions principales). Dans l'objectif de mieux comprendre en quoi les zones de forces des coalitions majeures se différencient, la troisième partie analyse le lien entre les résultats du scrutin et la dimension sociodémographique des territoires avant d'ouvrir des nouvelles pistes de recherche.

\section{Le contexte}

5 Le vote du 4 mars marque, sous plusieurs aspects, une rupture importante avec le passé. Pour comprendre en quoi la géographie politique italienne de 2018 se distancierait de celle des scrutins précédents, une brève histoire de la géographie électorale du Belpaese s'impose. Bien que stable, quelques modifications importantes ont eu lieu, s'accompagnant souvent de reformes de la loi électorale. Le scrutin du 4 mars ne fait pas exception car il suit une importante réforme du mode de scrutin. Le cadre législatif dans lequel le vote du 4 mars s'est déroulé a des incidences sur la " géographie » du vote, au moins dans le choix du niveau d'analyse, car la nouvelle loi électorale, dite Rosatellum, réintroduit une partie de scrutin majoritaire dans un système qui a toujours été proportionnel, hormis la parenthèse de trois élections entre 1994 et 2001.

\section{La géographie politique de l'Italie républicaine : une brève histoire}

6 Le système politique italien a longtemps été caractérisé par un pluralisme polarisé (Sartori, 1966) qui voyait s'opposer deux grands partis de masse, le Partito Comunista Italiano (Parti communiste Italien - PCI) et la Democrazia Cristiana (Démocratie 
Chrétienne - DC - parti centriste au gouvernement pendant 40 ans). Cette période, que l'on appelle de "Première République " (1948-1994), est à corréler à une géographie électorale bien connue (Galli et Capecchi, 1968). Il était alors possible d'identifier en Italie deux principales cultures politiques, une "blanche» et une "rouge", selon la préférence de vote soit pour la DC, soit pour les forces socialistes-communistes, en particulier pour le PCI (illustration 1). Ces cultures émergeaient de la façon dont était organisée la relation entre politique et société dans des territoires géographiquement définis. Dans plusieurs zones du pays, le monopole électoral domine (Trigilia, 1981; Mannheimer et Sani, 1987): d'un côté, les zones «blanches », qui votaient pour les centristes de la DC, situées dans le nord-est du pays et caractérisées par un réseau dense d'associations catholiques; de l'autre côté, les bastions des partis socialistescommunistes, des zones à fort taux de syndicalisation et où les associations (surtout celles culturelles et récréatives) étaient monopolisées par le PCI. Ces régions de l'Italie centrale (Emilie-Romagne, Toscane, Marches, Ombrie) sont surnommées regioni rosse (régions rouges) pour la stabilité de leur vote à gauche.

7 La limite territoriale Nord-Sud est également fondamentale quant à l'interprétation du comportement électoral de cette période. Cette division identifiait (et continue à le faire) des différences territoriales importantes en termes de développement économique, de système productif et de degré d'urbanisation. Dans les grands centres urbains, en particulier ceux du nord-ouest fordiste, la masse des travailleurs industriels était plus encline à voter pour les partis de gauche alors que dans le sud agricole et frappé par un chômage endémique, c'était la DC qui dominait.

Cet équilibre spatial commence à entrer en crise vers la fin des années 1980, lorsque trois facteurs interagissent : les changements internationaux (chute du mur de Berlin), les scandales de corruption du parti dominant (Diamanti 2009; Anderlini 2009) mais aussi, bien que moins étudiée, l'impossibilité pour les partis au gouvernement de satisfaire les intérêts divergents des catégories sociales composant leur propre électorat dans le système européen en formation. Ainsi, le «blanc » du DC est remplacé par le « vert » de la Lega Nord (Ligue du Nord), parti autonomiste qui évoluera pendant les années 1980-1990 vers l'extrême droite (Ignazi 1996), et le «bleu » de Forza Italia (Force Italie), le parti personnel de Silvio Berlusconi. Les dynamiques territoriales sont réinterprétées par ces nouveaux acteurs (Diamanti 2009) : d'une part, la Ligue du Nord s'enracine dans l'Italie septentrionale en devenant le représentant des territoires ruraux de la Lombardie, de la Vénétie et d'une partie du Piémont; de l'autre, grâce à son réseau de médias, Berlusconi organise son parti au-dessus de la dynamique territoriale et au-delà des fractures économiques et sociales locales. À gauche, les bastions rouges semblent d'abord résister. Malgré la dissolution du PCI dans un nouveau parti (le Partito Democratico della Sinistra, Parti Démocrate de la Gauche - PDS), cette fois social-démocrate, les électeurs de ces zones lui restent fidèles. C'est donc ce bipolarisme centre-gauche/centre-droit qui caractérisera la Deuxième République et nous assisterons à une alternance entre ces deux pôles au pouvoir jusqu'à la crise économique de 2008 qui, du moins du point de vue chronologique, marque la fin de cette période sans que l'on soit certain de l'avènement d'une Troisième République (Diamanti, 2012 ; Bull et Pasquino, 2018).

9 Il n'est pas étonnant que la crise économique ait eu un impact différencié sur le plan géographique. De même, la récente et légère reprise a été ressentie de manière profondément inégale le long de la Botte et par les différents blocs sociaux (ASviS, 
2017). Les ondes de ces chocs économiques et sociaux se sont concrétisées dans la dernière élection, provoquant une nouvelle tempête politique que nous pouvons considérer comme la troisième révolution électorale en cinq ans avec l'élection législative de 2013 et celle des européennes de 2014 (Chiaramonte et De Sio, 2014, De Sio et al., 2014). En 2013, ce sont les débuts extraordinaires d'un nouveau concurrent national, le Mouvement 5 Etoiles (M5S) : jamais, dans des élections non fondatrices d'une démocratie occidentale, une force politique n'a atteint un résultat aussi significatif lors de sa première compétition électorale (Cataldi et Emanuele, 2013). L'émergence de cette force a eu des répercussions importantes sur la géographie politique italienne et les résultats du scrutin du 4 mars 2018 semblent signifier que ces changements ne sont pas encore terminés. Effectivement, par rapport à 2013, la frontière Nord-Sud devient plus nette : le vote méridional privilégie le M5S, pendant que la Ligue (qui abandonne la dénomination « du Nord " pour devenir parti national) redevient désormais le premier parti de l'Italie septentrionale 20 ans après le scrutin de 1996.

10 Avant d'entrer dans le détail des résultats du scrutin, il est essentiel de présenter le système électoral en place lors du scrutin du 4 mars. Même si elles ne sont pas majeures, ce système a probablement eu des conséquences sur les résultats (Emanuele et Vassallo, 2018) et, surtout, sa genèse témoigne de la situation chaotique qui caractérise le système italien en 2018.

\section{Les règles du jeu}

11 Depuis fin 2017, suite à l'approbation de la loi Rosato ou Rosatellum (du nom de son défenseur, le député du PD Ettore Rosato), le système électoral italien se présente comme un système mixte mêlant des éléments majoritaires et proportionnels. En effet, il ne s'agit pas d'un système majoritaire pur, étant donné que la compétition entre candidats qui définit la composition des assemblées électives ne se fait pas exclusivement dans les circonscriptions uninominales; mais l'attribution des sièges ne se fait pas non plus de manière proportionnelle, modèle qui a caractérisé une bonne partie de l'histoire italienne.

12 La loi prévoit que $64 \%$ des sièges (386 sièges à la Chambre des Députés, 193 au Sénat) soient attribués proportionnellement, selon les résultats obtenus par les coalitions de liste au niveau national, tandis que les $34 \%$ restants sont réservés aux candidats ayant reçu le plus de votes dans 232 circonscriptions uninominales (116 au Sénat) dans lesquelles le scrutin se déroule à tour unique. Enfin, 12 sièges ( 6 au Sénat) sont réservés aux candidats élus par les Italiens résidant à l'étranger dont le vote est organisé par une loi différente ${ }^{1}$.

Les candidats de la partie uninominale et les listes de la partie proportionnelle sont liés, chaque candidat uninominal étant soutenu officiellement par une liste ou une coalition de listes qui participe à la compétition proportionnelle. Il est à noter que le vote se déroule à bulletin de vote unique. Sans rentrer dans les détails techniques du vote, le principe est que l'électeur choisit la liste pour laquelle il souhaite voter dans la partie proportionnelle et, automatiquement, son vote est transféré au candidat uninominal soutenu par la liste (ou par la coalition dont fait partie la liste). Ce système permet et, d'une certaine manière, favorise la création d'alliances entre listes différentes : selon la fameuse loi de Duverger (1951), la présence d'une large partie proportionnelle 
encourage la prolifération de listes alors que la partie uninominale (où "winner takes all», «le gagnant prend tout») pousse ces listes à s'allier. Pour la partie proportionnelle, un seuil minimum de $3 \%$ est fixé pour l'obtention de sièges. Dans le cas de coalitions de listes, les sièges sont attribués selon le score total de la coalition, y compris les listes ayant obtenu moins de $3 \%$ mais plus que $1 \%$ des suffrages ${ }^{2}$. Les listes proportionnelles sont bien des listes bloquées à partir desquelles les élus seront identifiés : dans la partie proportionnelle, l'électeur ne peut donc pas choisir pour quel candidat de la liste voter, mais juste pour quelle liste.

Le Rosatellum est le résultat de vingt ans de transformations du système électoral italien. La Première République était caractérisée par son mode de scrutin proportionnel pur mais ce système a été remis en cause suite aux résultats d'un référendum populaire de 1993 qui, en modifiant des parties substantielles de la loi, a obligé les législateurs à adopter un système partiellement majoritaire. C'est donc depuis l'élection législative de 1994 que les Italiens votent selon la loi dite Mattarellum, un système majoritaire avec un quota proportionnel.

Le Mattarellum a résisté pendant trois scrutins $(1994,1996,2001)$ puis a été remplacé par la loi Calderoli (du nom de son rapporteur). Cette loi, proposée et adoptée par le gouvernement de centre-droit, représentait un retour substantiel à la proportionnelle avec un élément de majority-assuring (Chiaromonte et D'Alimonte, 2018): une prime destinée à assurer la gouvernabilité en assurant à la coalition gagnante 340 sièges à la Chambre sur les 630. Après trois scrutins $(2006,2008,2013)$, jugeant cette prime excessive et créatrice de distorsion par rapport à la volonté des électeurs, la Cour Constitutionnelle est intervenue en 2014. Pour se conformer à la décision de la Cour, le gouvernement Renzi a donc proposé d'attribuer cette prime à travers l'institution d'un second tour entre les deux forces ayant obtenu le plus de votes. La Cour est intervenue de nouveau en 2017 en rejetant la nouvelle loi, qui est donc restée inutilisée.

Dans une situation instable, suite à l'abrogation (partielle) de deux lois électorales de la part de la Cour Constitutionnelle, les forces politiques ont donc essayé de trouver un accord pour une troisième loi, cette fois-ci respectueuse du dicté constitutionnel. Dans ce contexte, le PD tentait de s'opposer à la dispersion des voix de gauche, dans la logique du « vote utile » contre le centre-droit et le(s) populisme(s) représentés, dans la communication du PD, par la Lige et le M5S. De plus, pendant la période du Mattarellum, le centre-gauche a obtenu de meilleurs résultats dans la partie majoritaire du scrutin, raison pour laquelle la proposition initiale du centre-gauche était celle de réinstaurer in toto le Mattarellum (Chiaromonte et D'Alimonte, 2018). Le centre-droit, pour sa part, visait plutôt à maintenir l'instrument de la coalition, aussi pour permettre à la Ligue de Salvini et à la FI de Berlusconi de « se peser » dans les urnes, pour comprendre laquelle des deux listes avaient le plus de soutien. À cela, on peut ajouter l'espoir de ces deux forces de contenir le résultat du M5S, force qui rejette toute forme d'alliance électorale et qui était considérée comme «faible » en termes de qualité des candidats à présenter 3. Le Rosatellum a donc été le résultat d'un compromis entre centre-gauche et centredroit pour résoudre une situation " d'illégalité » engendrée par les deux lois électorales rejetées par la Cour Constitutionnelle.

17 La principale nouveauté introduite par le Rosatellum est le retour des circonscriptions uninominales. Moins larges qu'une province, ces circonscriptions ont été déterminées par le Gouvernement en collaboration avec le Parlement et l'Institut National de 
Statistique (ISTAT) en respectant des critères d'homogénéité géographique et sociale (Alleva, 2018). C'est donc là le niveau d'analyse des résultats électoraux.

\section{La géographie politique italienne après le 4 mars}

18 Le scrutin italien du 4 mars, le premier avec la nouvelle loi électorale dite Rosatellum, a présenté des éléments de rupture importants avec le passé, alors même que le vote de 2013 était déjà apparu comme un "tsunami » (Cataldi et Emanuele 2013). Nous allons étudier ces changements en se concentrant tout d'abord sur la participation puis en observant les résultats des coalitions principales.

\section{Des différences territoriales importantes dans une dynamique de déclin de la participation électorale}

Historiquement, l'Italie a longtemps été caractérisée par un taux de participation élevé, supérieur à $90 \%$ jusqu'à l'élection de 1979. Cette forte participation électorale n'était pas géographiquement homogène, un écart important ayant toujours été manifeste entre le Sud et le Centre-Nord. La stabilité de la participation a fait en sorte que, comme le rappelle Cerruto (2012), l'étude du taux d'abstention n'avait pas d'intérêt scientifique ou politique. La situation a commencé à changer au début des années 80 , lorsque le taux de participation a commencé à baisser. D'un phénomène résiduel, l'abstention semble être devenue une caractéristique structurelle du système démocratique italien, signal d'une crise plus générale.

20 L'analyse de l'élection du 4 mars 2018 doit donc d'abord prendre en considération les variations du taux de participation, en identifiant en particulier trois éléments qui ont caractérisé le scrutin: (i) une confirmation de la tendance au déclin du taux de participation, (ii) un ralentissement de ce déclin par rapport aux années précédentes et (iii) des différences territoriales importantes. Le taux final de participation a en effet été attesté à 72,6\% des ayants droit, en baisse de 2,6 points par rapport à 2013. Si nous élargissons le propos aux votes blancs ou nuls, la participation diminue encore, atteignant $70,6 \% \%$, le plus bas de l'histoire italienne. Ce chiffre confirme une tendance à long terme de désaffection des électeurs italiens. En même temps, il existe des signes d'ajustement : la baisse est plus modeste que celle enregistrée entre 2008 et 2013 (De Lucia et Cataldi, 2013). Les cartes présentées (illustrations 2 et 3) montrent respectivement la répartition de la participation et sa variation par rapport au dernier tour électoral. Comme les années précédentes, la participation n'est pas homogène sur le territoire, avec des pics en particulier dans le Nord de l'Italie. Au premier abord, nous retrouvons ici une constante de la géographie politique italienne : les électeurs du Mezzogiorno, des zones caractérisées par un taux de chômage plus élevé et, en général, par de plus grandes difficultés économiques, tendent à moins se présenter aux urnes que dans le reste du pays (Rolfe, 2012).

21 Malgré la persistance de différences géographiques importantes, le niveau de participation apparaît plus territorialement homogène qu'il y a cinq ans. Effectivement, une partie importante du bassin historique de la gauche italienne, les "regioni rosse» (Emilia-Romagna, Marche, Umbria, Toscana) situées au centre du pays, connaissent une baisse significative du taux de participation, autrefois parmi les plus élevés du pays. A contrario, sur les 13 provinces qui voient leur taux de participation augmenter, huit se 
situent dans le Mezzogiorno. Les cinq restantes sont situées dans l'Italie septentrionale, à la frontière entre Italie et Suisse : il s'agit d'une partie du pays caractérisée par des villes de taille réduite et d'un bassin électoral important pour la Ligue. Ces données préliminaires fournissaient déjà, avant même le dépouillement, des indices sur la dynamique des résultats identifiant un possible perdant: le centre-gauche et en particulier le PD de Matteo Renzi, qui n'apparaît pas capable de motiver et mobiliser son propre électorat historique.

\section{L'Italie de 2018 : un système tripolaire mais imparfait}

Comme expliqué dans la partie précédente, suite à la réforme de la loi électorale en novembre 2017, la péninsule a été divisée en 232 circonscriptions uninominales. Si l'on regarde la carte des gagnants dans les circonscriptions (illustrations 4 et 5) et celle des partis ayant obtenu le plus de votes (illustrations 6 et 7), l'Italie est divisée en trois, avec deux coalitions majeures (le centre-droit et le M5S) et une troisième, le centregauche, plus petite et qui ne paraît pas en condition d'inquiéter ses adversaires: le système apparaît donc inégalement tripolaire.

\section{Le centre-droit italien et l'affirmation (définitive ?) de la Ligue} coalition de centre-droit qui a obtenu le plus de voix le 4 mars dernier. Son principal bassin électoral a été le Nord : le centre-droit arrive à y dépasser le seuil de $50 \%$ dans 20 circonscriptions, toutes situées entre la Lombardie et la Vénétie, régions historiques d'enracinement de la Ligue. L'Italie septentrionale se colore donc de bleu en raison du score de la Ligue. A l'opposé, dans le Sud, longtemps zone de force de Berlusconi et du FI, le centre-droit n'arrive en tête que de deux circonscriptions. principaux de la coalition. D'un côté, FI, parti de l'ancien Premier Ministre et leader historique du centre-droit Silvio Berlusconi, apparaît comme le grand perdant d'une coalition qui, elle, gagne largement des voix par rapport à 2013 (illustrations 8 et 9). Le déclin de FI par rapport au résultat du Popolo della Libertà en 2013 est homogène dans tout le pays : dans neuf régions, FI remporte moins de $60 \%$ des voix par rapport au scrutin précèdent. La diminution est légèrement plus sensible dans le Nord-Est, avec un degré de " résistance " ${ }^{4}$ à peine supérieur à $50 \%$ en Vénétie et Frioul-Vénétie Julienne, là où la concurrence avec la Ligue a été plus forte. Dans un contexte général de recul, le parti de Silvio Berlusconi se maintient davantage au Sud, avec un taux de résistance autour $67 \%$. Dans une circonscription comme celle de Gioia Tauro en Calabre, son résultat est même meilleur qu'il y a cinq ans, avec un solde positif de 1200 voix. Malgré un taux de résistance supérieur, le résultat du Sud reste décevant et, au niveau municipal, FI n'est en position dominante que dans 83 municipalités méridionales sur 1710.

De l'autre côté, la Ligue, parti historiquement «nordiste» qui arrivait à peine à recueillir des voix au Sud de la Toscane, est rentré dans une phase de transformation profonde sous la direction de Matteo Salvini avec l'intention de devenir un parti national. La disparition du mot « Nord » du nom même du parti ou le changement des slogans de campagne de "Prima il Nord " ("Le Nord d'abord») à "Prima gli italiani " («Les Italiens d'abord») en témoignent ${ }^{5}$. Le pari de «nationalisation» de Salvini 
apparait comme gagnant: en 2018, l'électorat de la Ligue apparait moins concentré qu'en 2013, comme le montre l'analyse du coefficient de variation ${ }^{6}$, plus de deux fois inférieur à celui de 2013. La carte de la répartition des voix (illustration 10) nous renvoie une Ligue non seulement capable de renforcer ses positions dans le Nord, mais qui a commencé sa descente vers le Sud, en particulier avec des scores très importants dans le centre du pays.

Ces forts scores dans le Mezzogiorno, jusqu'alors hostile, ont logiquement attiré l'attention des observateurs, mais le résultat dans le Nord mérite d'être souligné aussi. La Ligue atteint $33 \%$ des voix en Vénétie ( $11 \%$ en 2013 ) et $29 \%$ en Lombardie ( $13 \%$ en 2013) (illustration 11), ainsi que $25 \%$ dans le Piémont (un résultat cinq fois supérieur à celui obtenu en 2013) et $20 \%$ en Ligurie (huit fois plus qu'en 2013). Il s'agit de résultats historiquement élevés pour une élection législative. Le leadership de Salvini a donc su convaincre à la fois sa base historique et identitaire, qui se mobilise autour du thème de l'autonomie régionale des régions $\mathrm{du}$ nord, mais aussi de nouveaux votants, probablement anciens électeurs de Forza Italia.

Cela apparait encore plus clairement si nous élargissons la perspective au-delà de l'Italie du Nord: dans les circonscriptions du centre de l'Italie, la Ligue passe de $1 \%$ à $17 \%$ et réussit à dépasser $10 \%$ des suffrages dans la capitale, Rome, qui faisait pourtant l'objet de slogans virulents («Roma Ladrona», Rome la grande voleuse) de la part de la Ligue « du Nord » du passé. Dans l'Italie méridionale, la Ligue obtient des scores au-delà de $3 \%$ dans toutes les régions du Sud. À titre d'exemple, nous pouvons citer la ville de Lecce où la Ligue obtient plus que 3200 voix : ils n'étaient que 20 électeurs en 2013, soit une augmentation de plus de 160 fois. En Sicile, région polarisant les flux de réfugiés provenant de Lybie, et en particulier sur la côte méridionale, la plus exposée aux débarquements, la Ligue obtient des résultats non négligeables: $7 \%$ dans la circonscription de Gela et $6 \%$ dans celle de Agrigento. À Lampedusa, île symboliquement importante et véritable porte d'entrée en Europe, le parti de Salvini recueille $14 \%$ des voix en 2018 alors que la liste ne dépassait pas $0,15 \%$ en 2013. Il s'agit d'un résultat supérieur à celui que la Ligue remporte dans la circonscription centrale d'une grande ville du Nord comme Turin (13,7 \%), il était impensable jusqu'à il y a 5 ans. Bien que l'implantation soit encore inégale entre le Nord et le Sud, nous pouvons en conclure que, au moins pour l'instant, la cohabitation entre les deux âmes de la Ligue de Salvini - la nouvelle à projection nationale et celle historiquement liée aux demandes d'autonomie - réussit.

\section{L'hégémonie à 5 étoiles qui témoigne d'une nouvelle " question méridionale »}

Au Sud, c'est le jaune du M5S qui domine (illustration 12). Le parti "populiste », avec son message anti-casta, qui identifie dans la classe politique (et dans ses revenus) le premier ennemi (Ivaldi et al., 2017), recueille la majorité absolue des suffrages dans un tiers des circonscriptions méridionales et les candidats pentastellati arrivent en tête dans 70 des 73 circonscriptions du Sud. Au niveau municipal, le M5S est la liste ayant obtenu le plus de voix dans 1588 municipalités méridionales sur 1710.

L'augmentation par rapport à 2013 est frappante : au niveau national, la liste gagne plus de 2 millions de voix et 7 points en pourcentage. Mais cette croissance est loin d'être homogène sur l'ensemble du territoire national (illustration 13): elle se concentre dans le «Mezzogiorno ». Si l'on observe la percée d'un point de vue relatif, l'augmentation moyenne est égale à 20 points, avec un pic en Campanie $(+27,5$ points 
sur 2013) et dans la région de Naples, bassin historique pour le centre-droit berlusconien. Au Centre et au Nord, les candidats pentastellati remportent des résultats inférieurs et parfois décevants. C'est en particulier le cas de l'Italie septentrionale, où le M5S souffre de la concurrence de la Ligue : face à la sensible augmentation du score national des pentastellati, une perte de voix dans 58 circonscriptions sur 232 est observable, toutes au Nord de Rome. Dans l'Italie septentrionale, le M5S doit souvent se contenter de terminer troisième. Il n'est en position gagnante que dans trois circonscriptions, deux à Gênes et une à Collegno, dans la banlieue turinoise. À regarder de plus près le score des candidats pentastellati au Nord, il s'avère que c'est dans les banlieue des grandes villes que le M5S obtient ses meilleurs résultats, bien qu'ils n'arrivent pas à s'imposer comme première coalition. Il y a donc bien d'un côté le Mezzogiorno et sa question méridionale, déjà soulignée par Antonio Gramsci (1982) il y a un siècle, et, de l'autre côté, les quartiers périphériques du Nord.

\section{La défaite du centre-gauche et la transformation de l'électorat progressiste}

La dernière couleur que l'on peut retrouver sur la carte électorale italienne est le rouge qui identifie l'alliance de centre-gauche dont l'ancien Premier Ministre Matteo Renzi était le chef-de-file (illustration 14). L'alliance progressiste arrive encore à s'imposer dans des zones d'implantation historique tout au long des Apennins tosco-émiliens, mais cette zone apparait de plus en plus restreinte. À l'inverse, de nouvelles zones de force apparaissent: les circonscriptions centrales de certaines grandes villes (Rome, Milan et Turin) où les candidats du centre-gauche arrivent en tête. In fine, la défaite est cuisante pour la coalition de centre-gauche dont les candidats n'arrivent à gagner que 28 des 232 circonscriptions de la Chambre basse. Même dans les "regioni rosse", les candidats du PD et ses alliés se voient souvent dépassés et battus, en particulier par les candidats du centre-droit. Ce résultat est tellement mauvais que l'Istituto Cattaneo, qui avait inventé en 1968 la dénomination "regioni rosse», est arrivé à en certifier la disparition comme zone politiquement homogène (Valbruzzi 2018). Dans cette partie du pays, le PD perd 8,7 points par rapport aux élections de 2013 et l'alliance de centregauche n'arrive à s'imposer que dans 16 circonscriptions sur 40. C'est en EmilieRomagne que la perte est le plus remarquable : les démocrates n'arrivent à garder que 65 voix sur 100 obtenues en 2013 et, pour la première fois depuis la fin de la Seconde Guerre Mondiale, le centre-droit arrive en tête. La Toscane, région d'origine de Matteo Renzi, ancien maire de Florence, n'est pas épargnée par ce déclin, mais nous pouvons remarquer un degré de résistance supérieur. L'hémorragie est encore plus marquée au sud, où le centre-gauche perd en moyenne 8 points sur 2013 en n'obtenant que le $16 \%$ des suffrages (illustration 15).

31 Dans cette tendance globale, signalons des exceptions. Dans cinq circonscriptions, le PD arrive en effet à augmenter son score relatif par rapport à 2013 : il s'agit des deux circonscriptions centrales de Milan et de la circonscription centrale de Turin, plus les deux circonscriptions à majorité germanophone grâce à l'alliance avec le parti autonomiste de la minorité de langue allemande du Haut-Adige/Südtirol, la Südtiroler Volkspartei (Parti populaire sud-tyrolien - SVP). De même, les résultats du centre de Rome apparaissent très positifs; c'est là que l'alliance de centre-gauche présentait comme candidats à la partie uninominale des personnalités importantes de l'expérience gouvernementale de 2013-2018, c'est-à-dire le Premier Ministre sortant Paolo Gentiloni et la Ministre de la Fonction Publique Marianna Madia. 
32 Le score globalement négatif du centre-gauche italien cache donc des mutations territoriales, et donc sociales, majeures, que l'on peut effectivement retrouver dans les différentes analyses de sondages parues après le 4 mars (Valbruzzi et Vignati, 2018). Le centre-gauche parait vivre une profonde transformation qui l'amène à être le premier choix des classes aisées du pays, et le troisième des autres. Cette lecture apparait également valable lorsque nous regardons de plus près le contexte social qui caractérise les zones de force des différentes coalitions.

\section{Trois Italies pour trois électorats différents : les clivages sociaux à la loupe}

Dans la partie précédente, nous avons donné aux lecteurs des éléments généraux quant aux résultats de l'élection italienne du 4 mars 2018, en présentant la distribution géographique du soutien reçu par les trois coalitions majeures et sa variation par rapport à l'élection de 2013. La nouvelle carte de l'Italie ainsi dépeinte renvoie à des contextes socio-économiques très différents, qui donnent à comprendre les différents clivages structurant le système politique italien. Mais quelles sont les différences entre les "trois Italie» que nous venons d'identifier en termes sociodémographiques, à travers des variables telles le degré d'urbanisation, le niveau d'étude, le pourcentage de population étrangère, etc.?

\section{Un centre-gauche urbain, une Ligue forte dans les villes de petite taille et le M5S comme parti transversal}

La variation des scores selon la taille de la ville (tableaux 2 et 3) permet d'observer la manière dont le centre-droit et le centre-gauche sont à l'un l'opposé de l'autre. La coalition de Matteo Renzi est en difficulté dans les petites villes, où elle n'obtient que $20,7 \%$ des voix, mais les scores remontent avec l'augmentation du nombre d'habitants. Le résultat du centre-gauche dans les centres urbains de plus de 300000 habitants est ainsi très important. Si ce clivage a toujours existé dans la deuxième République, le centre-gauche de Renzi voit pourtant augmenter, depuis 2013, la distance entre les scores obtenus dans les contextes ruraux et ceux des zones urbaines, le degré de résistance étant plus élevé dans les grandes villes. Même à l'intérieur des zones urbaines, il faut signaler l'existence d'un clivage entre les centres-villes et leurs banlieues. C'est en particulier le cas dans trois villes majeures: Rome, Milan et Turin. Dans la capitale, le centre gauche passe d'un score maximum de $42,1 \%$ enregistré dans la circonscription de Trionfale, dans le centre historique, à un minimum de 19,9\% obtenu dans la circonscription de Fiumicino qui regroupe les quartiers de la banlieue méridionale ainsi que la ville de Fiumicino. La même dynamique est observable à Milan : le centre gauche culmine dans l'une des circonscriptions touchant le centreville (Porta Romana, $41,3 \%$ ) et n'obtient que 28,7 \% à Baggio, dans la périphérie orientale. Finalement, à Turin, le centre-gauche remporte le siège uninominal dans la circonscription du centre mais termine troisième dans la circonscription Nord de la ville, l'une des circonscriptions au taux de chômage le plus élevé, malgré le fait qu'elle se situe dans la partie riche du pays. Comme nous l'avons déjà souligné, on observe un basculement social de l'électorat progressiste. Ce basculement est encore plus marqué dans les grandes villes, au point que, par exemple, nous n'arrivons plus à retrouver de 
corrélation entre le vote pour le centre gauche en 2013 et celui pour l'alliance de Matteo Renzi en 2018 à l'échelle du bureau de vote dans la ville de Turin. Ce changement ne peut pas s'expliquer exclusivement par le rôle d'incumbent joué par le centre-gauche, dans une époque où de plus en plus les électeurs punissent les gouvernements sortants. Nos analyses montrent une "cohérence" entre le nouvel électorat progressiste et les modifications dans l'offre programmatique du centregauche (Cavallaro et al., 2018). En effet, le programme parlait principalement aux classes sociales habitant les ZTL (zone a traffico limitato - zones à trafic limité), donc les centres historiques et les quartiers « riches » des grands centres urbains, qui se sont mobilisées pour la coalition du PD. Cela n'a évidemment pas suffi.

Le centre-droit, lui, a parlé à son électorat "acquis » et présente une dynamique opposée. Effectivement, l'alliance entre Salvini et Berlusconi atteint un pic de 42,3\% dans les municipalités de moins de 5000 habitants, pour tomber ensuite au-dessous de $30,8 \%$ dans les grandes villes. Il est notable que cette tendance n'est pas identique à toute la coalition : la Ligue et FI montrent une évolution différenciée de leurs scores selon la taille des communes. Alors que ceux du parti de Salvini varient considérablement (avec un maximum de $22,8 \%$ dans les municipalités de moins de 5000 habitants et un minimum de 11,5\% dans les villes de plus de 300 000), les scores de FI semblent stables et arrivent même à surpasser la Ligue dans les grands centres urbains.

Le M5S semble, enfin, ne pas être affecté de manière significative par ce clivage. Alors que la liste de Di Maio obtient de meilleurs résultats dans les municipalités de taille moyenne, l'écart par rapport à la moyenne est inférieur à celui du centre-droit. Sans doute, cette dynamique peut être liée à l'excellent résultat de la liste dans le Mezzogiorno, qui compte un nombre plus élevé d'habitants dans de villes de taille moyenne.

\section{Pour aller plus loin : quelle relation entre caractéristiques sociodémographiques du territoire et le vote?}

37 Nous nous proposons d'étudier la relation entre les caractéristiques sociodémographiques du territoire et les préférences politiques en regardant le niveau de corrélation entre ces deux variables au niveau de la circonscription électorale. Nous choisissons ce niveau d'analyse plutôt que les provinces pour deux raisons: premièrement, le découpage des circonscriptions est fait de telle sorte que ces entités soient assez similaires en nombre d'habitants, ce qui n'est pas le cas pour les provinces. Deuxièmement, dans chaque circonscription, l'offre politique se présentait comme homogène: dans chaque circonscription les électeurs retrouvaient, pour chaque coalition, le même candidat au siège uninominal, alors que les provinces pouvaient être divisés en plusieurs circonscriptions, mélangeant donc des candidats différents.

Le tableau 4 montre donc le coefficient de corrélation, aussi dit " $r$ de Pearson », entre les variables proposées par ISTAT et le pourcentage obtenu par les listes principales dans les 231 circonscriptions électorales incluses dans notre analyse. Les données confirment nos affirmations précédentes: le centre-gauche apparait bénéficier d'une certaine polarisation urbaine. En revanche, cela joue de manière différente entre les alliés du PD. La liste dont le score est le plus étroitement corrélé à la densité de population est +Europa (+EU), liste menée par l'ancienne Commissaire Européen Emma 
Bonino et caractérisée par son fort européisme. Aussi, la corrélation est très nette avec le taux d'emploi, ainsi qu'avec le pourcentage de population étrangère.

La situation que nous observons lorsqu'on s'intéresse au M5S est toute différente : le vote pour les pentastellati montre une corrélation positive avec le taux d'analphabétisme ainsi qu'avec l'indice de vulnérabilité sociale, et négative avec le taux d'emploi. Les deux mouvements apparaissent donc opposés en termes de caractéristiques de leurs territoires d'ancrage: un centre-gauche fort dans les zones économiquement plus dynamiques et à capital culturel plus élevé par rapport à un mouvement M5S dont les soutiens augmentent dans les territoires plus délaissés.

La Ligue, parti habituellement considéré comme populiste (Albertazzi et McDonnell, 2015), se comporte de manière plus similaire au centre-gauche qu'au M5S : c'est FI qui voit son score varier en fonction des variables sociodémographiques plus similaires aux pentastellati. Sans doute, la division nord-sud a-t-elle une forte influence : la Ligue étant forte dans l'Italie septentrionale, ses scores nous apparaissent liés à toutes les caractéristiques de cette zone du pays. Sur ce point, une recherche plus approfondie serait nécessaire, passant des méthodes uni-variées aux méthodes multivariées pour vérifier la significativité de ces corrélations. En tout cas, il semblerait à ce stade que les deux partis " populistes ", grands gagnants de l'élection du 4 mars, n'apparaissent pas représenter les mêmes territoires, ce qui peut indiquer une différence aussi en termes de caractéristiques sociales de l'électorat ainsi que de demande politique. Le M5S est particulièrement fort dans les zones qui souffrent des migrations internes, dans les territoires fragiles en termes socioéconomique et au faible capital culturel. La Ligue, elle, est plus représentée dans des zones qui sont généralement plus riches et où le niveau d'éducation est en moyenne plus élevé, même sans toucher l'excellence. Finalement, les zones avec les taux de diplômés les plus élevés, les grands centres urbains, deviennent des bastions du centre-gauche.

\section{Conclusion}

41 Dans cet article nous avons analysé les résultats de l'élection italienne du 4 mars 2018. Après avoir présenté le contexte politique ainsi que la nouvelle loi électorale, nous nous sommes tout d'abord focalisés sur la participation: dans cette élection législative caractérisée par le plus faible taux de participation de l'histoire républicaine, l'augmentation de l'abstention est générale. Ainsi, l'Italie d'aujourd'hui, malgré la persistance de différences territoriales entre Nord et Sud, est plus homogène que dans le passé en termes de taux de participation.

Elle est, en revanche, très hétérogène lorsqu'on parle des préférences des électeurs. En citant Diamanti (2015), nous avons donc parlé de l'Italie comme d'un système politique tripolaire mais imparfait avec deux pôles majeurs et compétitifs (centre-droit et M5S) et un pôle, le centre-gauche, qui nous apparait loin des deux autres en termes de résultats. Pour mieux comprendre les caractéristiques territoriales de ces trois pôles, nous avons observé la relation entre distribution des préférences électorales et distribution des variables sociales au niveau des circonscriptions uninominales. Ainsi, si l'on regarde la distribution géographique du vote pour identifier quels facteurs locaux, en première analyse, favorisent le vote pour l'une des coalitions, trois éléments principaux susceptibles d'être approfondis dans les études à venir sont identifiables :

1. La Ligue obtient ses meilleurs scores dans les nombreuses communes de petites et 
moyennes dimensions du Nord, là où le taux d'occupation est élevé, les habitants plus éduqués que la moyenne nationale (mais peut-être moins que la moyenne du Nord) et où le taux d'immigration étrangère se situe à un niveau relativement haut.

2. Le centre-gauche est plus présent dans les grandes villes, notamment dans les quartiers caractérisés par un niveau d'étude plus élevé mais aussi dans les territoires caractérisés par la présence d'une population plus âgée. Le PD connait un changement important dans la nature de son électorat et il se retrouve avec un score historiquement bas; seule "véritable " opposition parlementaire au gouvernement M5S - Ligue, il se présente comme le parti de la gouvernabilité responsable contre le populisme. Ce message, dans un pays qui peine à retrouver les niveaux d'emploi d'avant la crise, ne semble pénétrer que dans les milieux le plus aisés.

3. Le M5S et FI vont main dans la main dans les régions du Sud, économiquement déprimées et sources historiques de l'émigration interne, présentant les taux d'analphabétisme les plus élevés ainsi qu'une fragilité sociale marquée.

Géographiquement les deux partis « anti-establishment » qui sont actuellement alliés et au gouvernement, après une campagne électorale menée l'un contre l'autre, sont l'expression de territoires aux caractéristiques différentes et presque opposées. Comme l'ont montré d'autres études menées à partir des données d'enquêtes (Cavallaro et al., 2018), cette première analyse de la distribution territoriale du vote met en évidence la possible fragilité et les contradictions entre l'électorat de la Ligue et celui pentastellato. C'est sur la capacité des deux forces politiques à réaliser la synthèse entre des mondes aussi différents (le nord des petites villes industrielles ainsi que les périphéries des grandes métropoles septentrionales pour la Ligue, les zones les plus délaissées du Sud pour le M5S) qui se joue donc le grand défi du gouvernement « populiste » italien. 
Illustration 1 - Liste ayant obtenu le plus de votes aux élections au début et à la fin de la lère République par province (1948 et 1992)

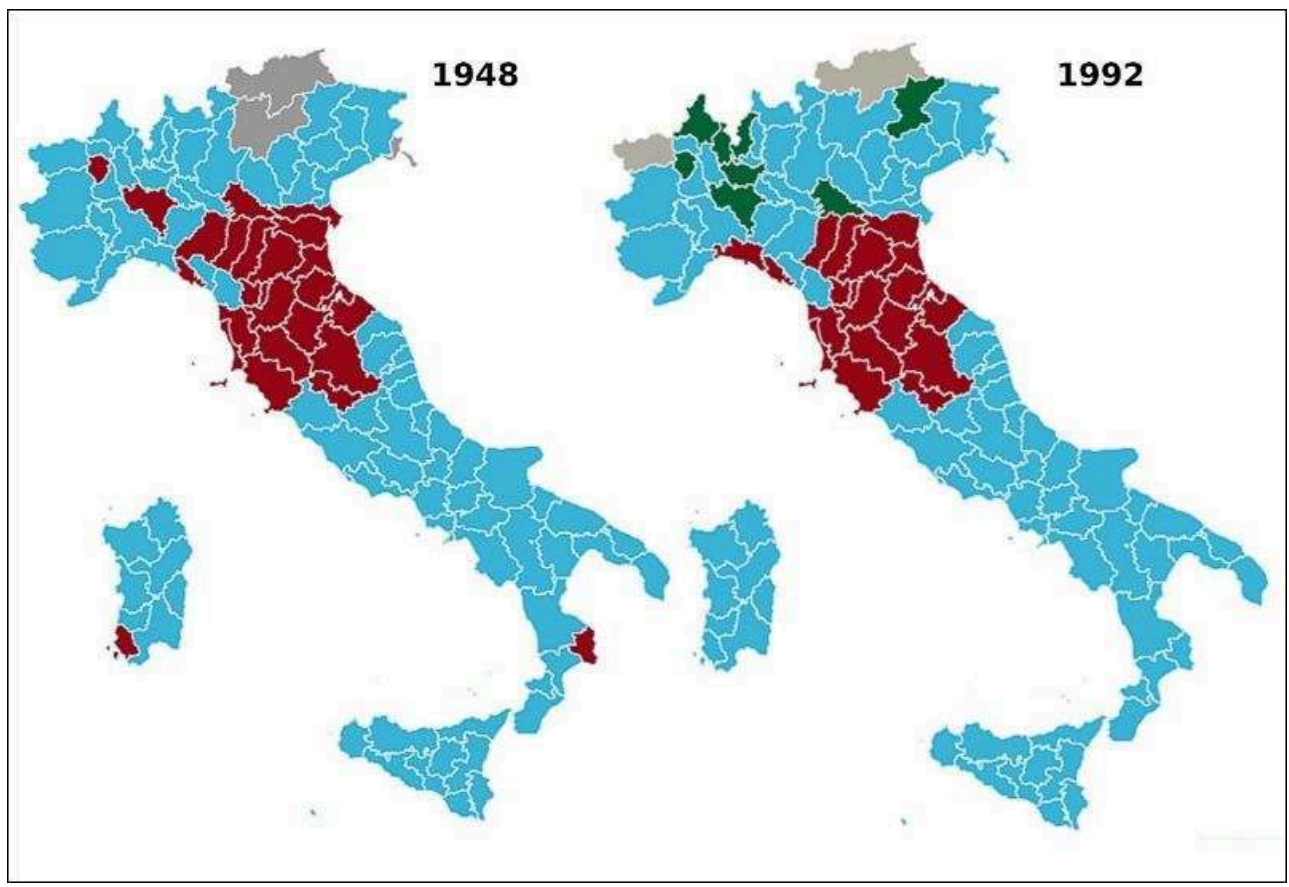

En bleu : la DC.

En rouge le Front Populaire (1948), qui regroupait les forces sociales-communistes, et le PDS (1992). En vert la Ligue.

Source : Calculs Quorum/YouTrend sur données du Ministère de l'Intérieur.

Illustration 2 - Distribution de la participation par province (élection législative 2018)

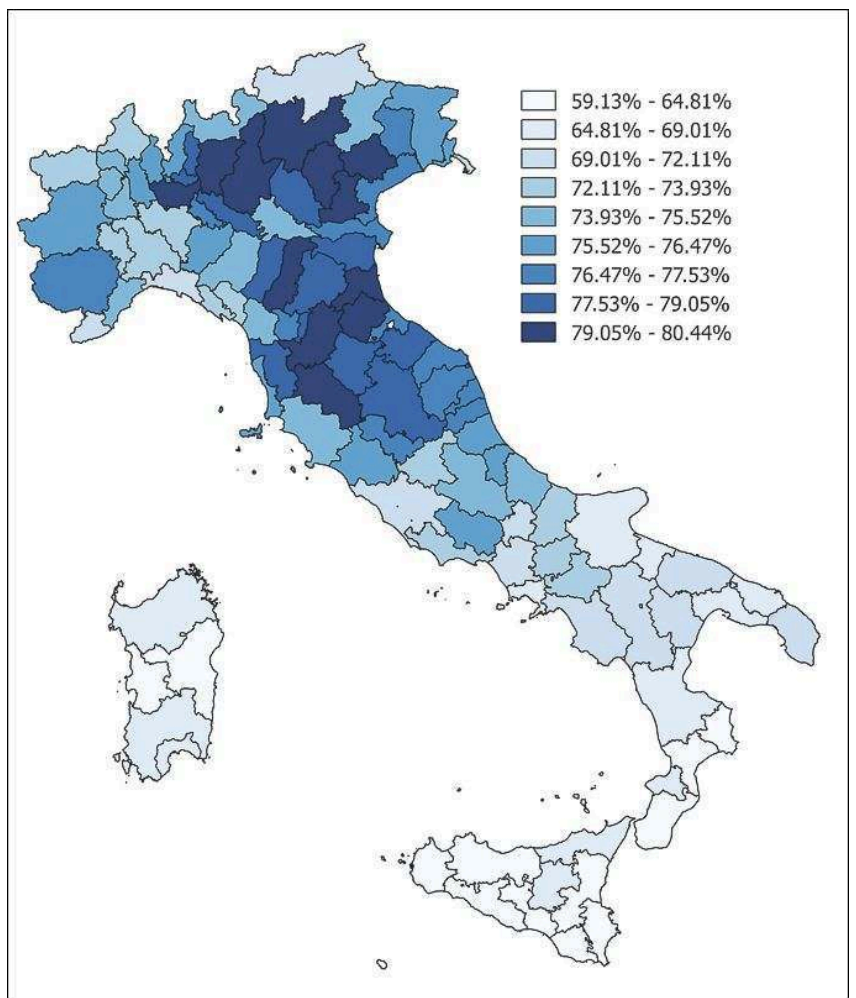

Source : Calculs Quorum/YouTrend sur données du Ministère de l'Intérieur. 
Illustration 3 - Variation de la participation 2018-2013 par province

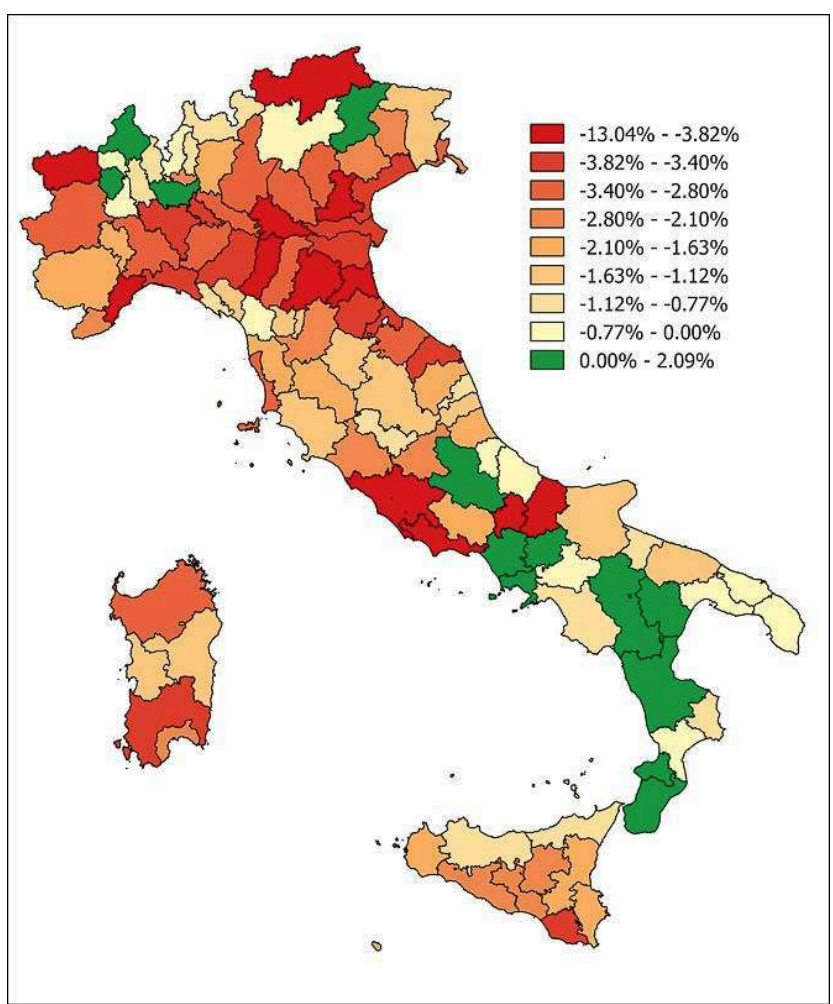

Source : Calculs Quorum/YouTrend sur données du Ministére de l'Intérieur. 
Illustration 4 - Coalition gagnante par circonscription uninominale (Chambre basse, élection législative 2018)

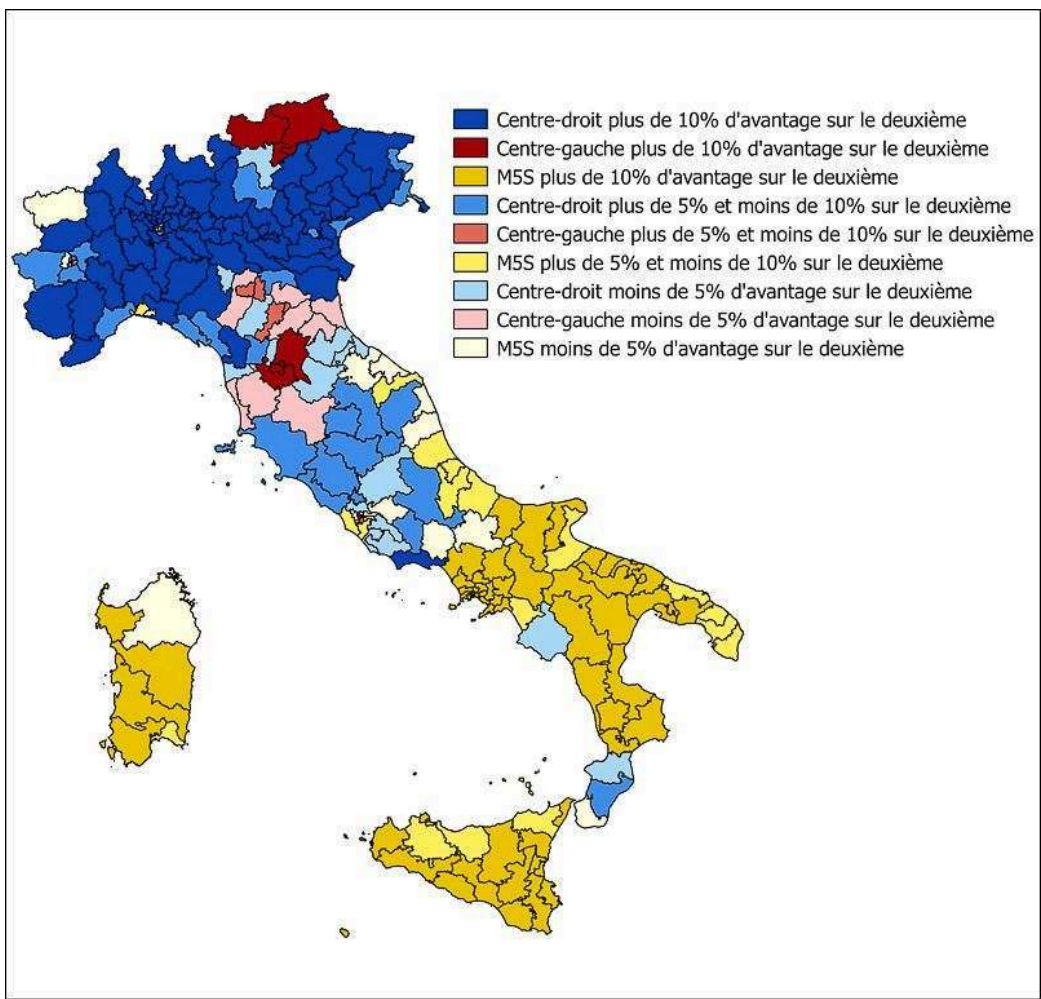

Source : Calculs Quorum/YouTrend sur données du Ministère de l'Intérieur. 
Illustration 5 - Coalition gagnante par circonscription uninominale (Chambre basse, élection législative 2018) - Carte en anamorphose

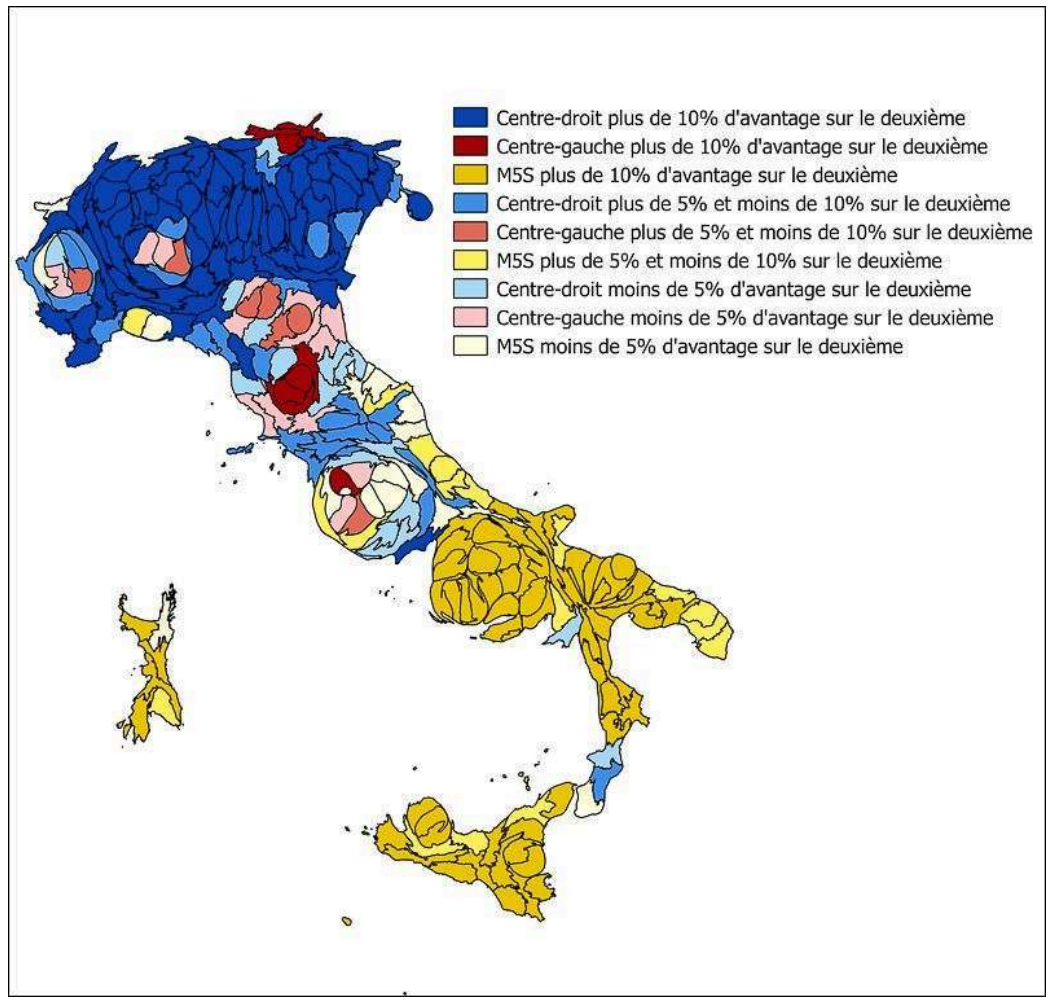

Anamorphose par population de la circonscription lors du recensement de 2011. Source : Calculs Quorum/YouTrend sur données du Ministère de l'Intérieur. 
Illustration 6 - Liste ayant eu le plus de votes dans la partie proportionnelle par circonscription (Chambre basse, élection législative 2018)

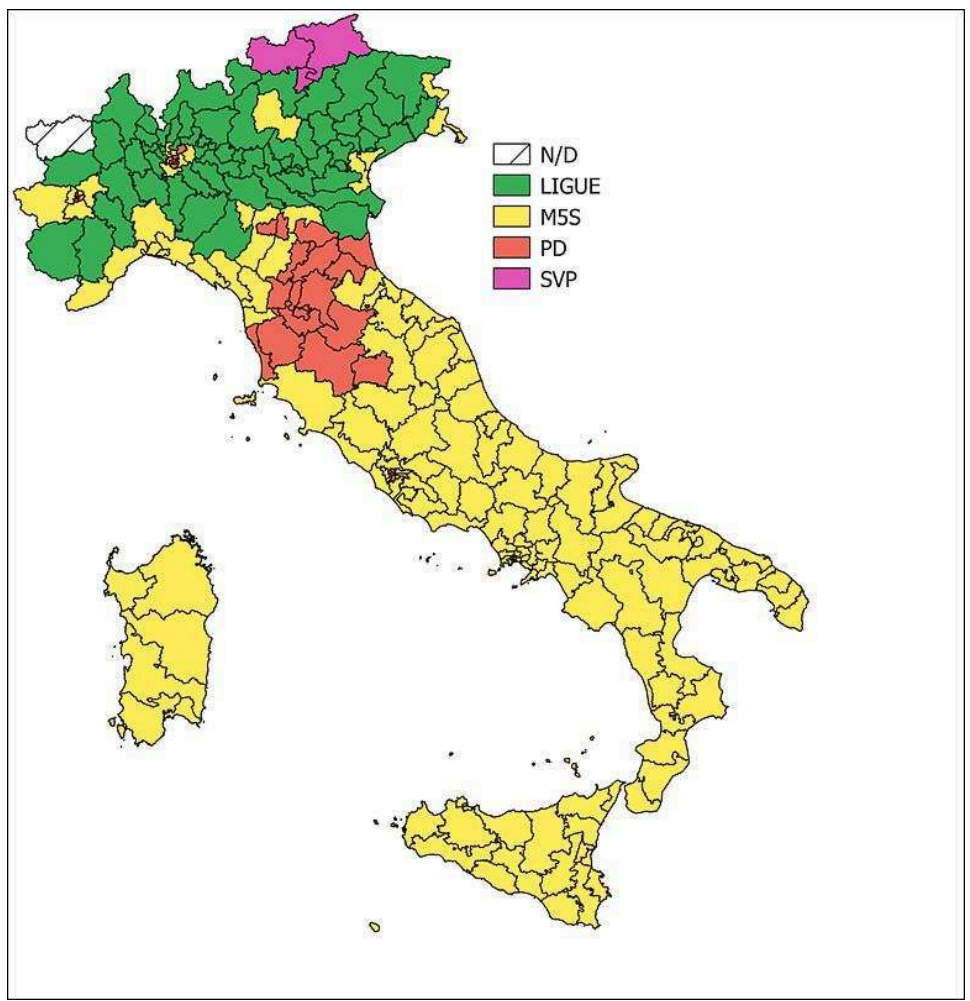

Source : Calculs Quorum/YouTrend sur données du Ministère de l'Intérieur. 
Illustration 7 - Liste ayant eu le plus de votes dans la partie proportionnelle par circonscription (Chambre basse, élection législative 2018) - Carte en anamorphose

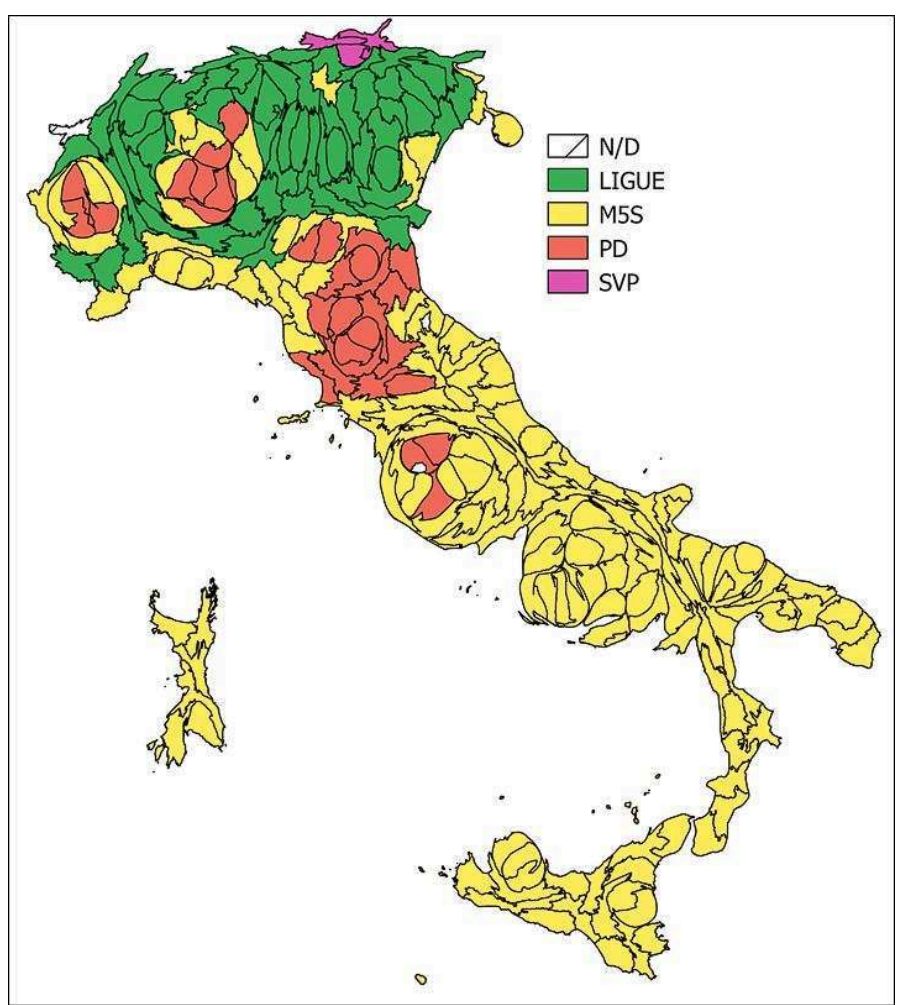

Anamorphose par population de la circonscription lors du recensement de 2011.

Source : Calculs Quorum/YouTrend sur données du Ministère de l'Intérieur. 
Illustration 8 - Distribution du score de FI par circonscription (Chambre Basse, élection législative 2018)

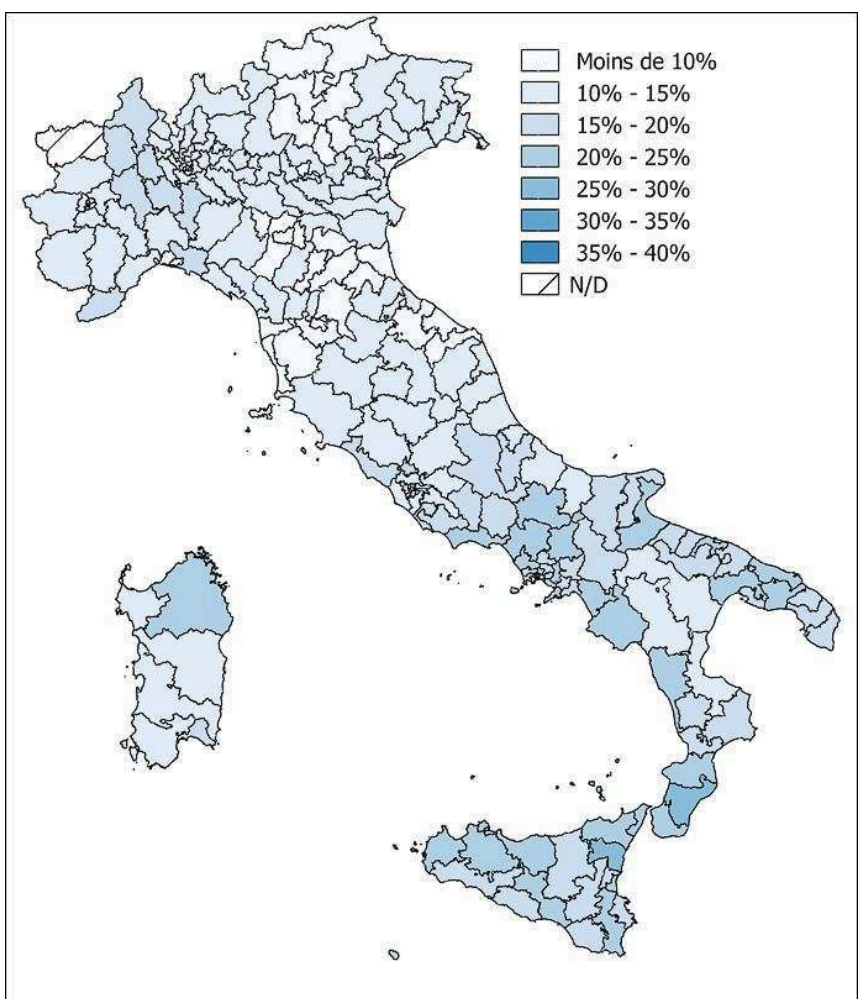

Source : Calculs Quorum/YouTrend sur données du Ministère de l'Intérieur. 
Illustration 9 - Variation du pourcentage de voix obtenu par FI par circonscription (Chambre basse, élection législative 2018)

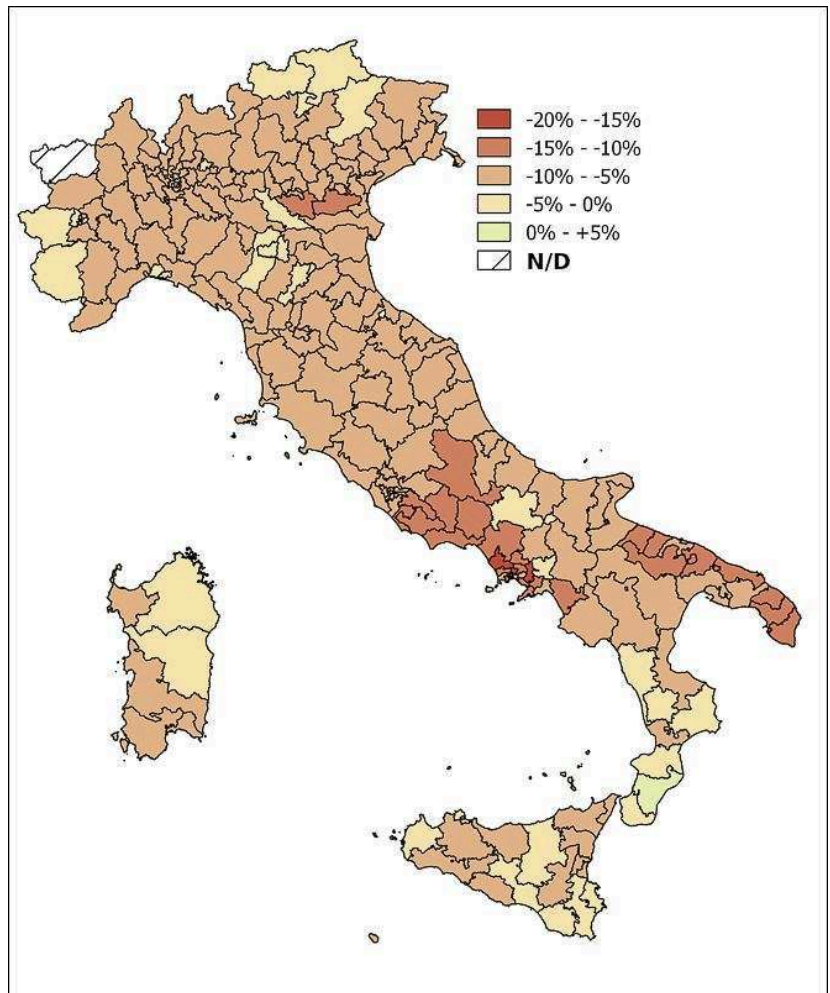

Source : Calculs Quorum/YouTrend sur données du Ministère de l'Intérieur. 
Illustration 10 - Distribution du score de la Ligue par circonscription (Chambre Basse, élection législative 2018)

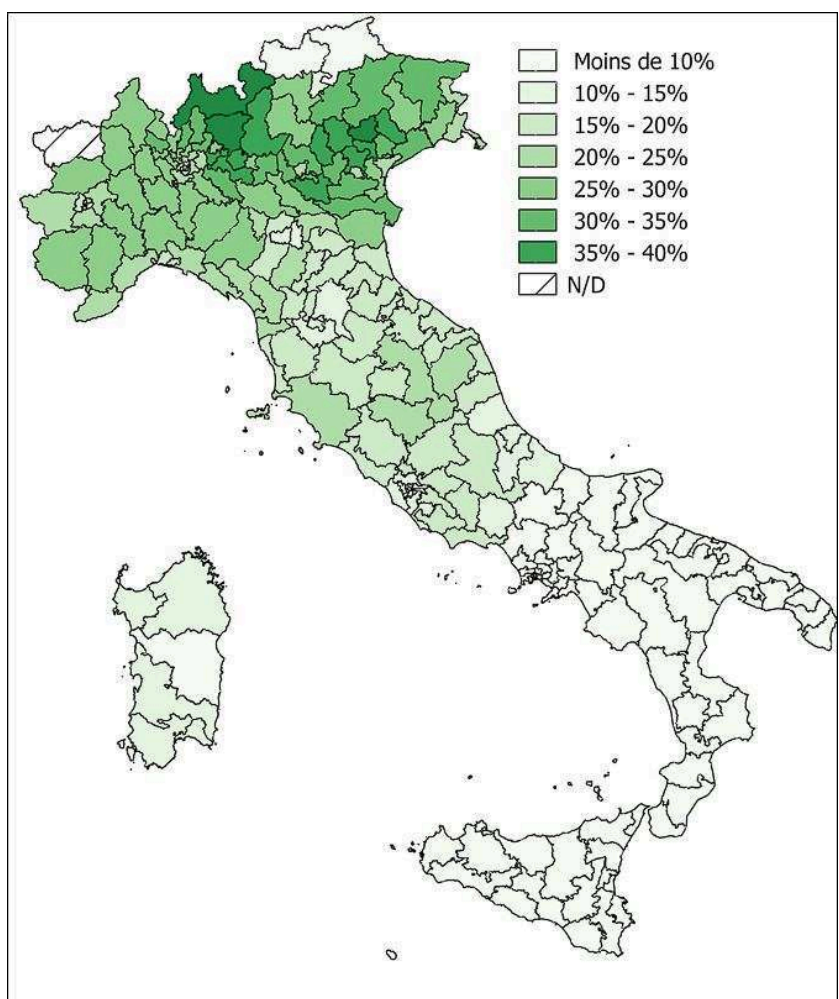

Source : Calculs Quorum/YouTrend sur données du Ministère de l'Intérieur. 
Illustration 11 - Variation du pourcentage de voix obtenu par la Ligue par circonscription (Chambre basse, élection législative 2018)

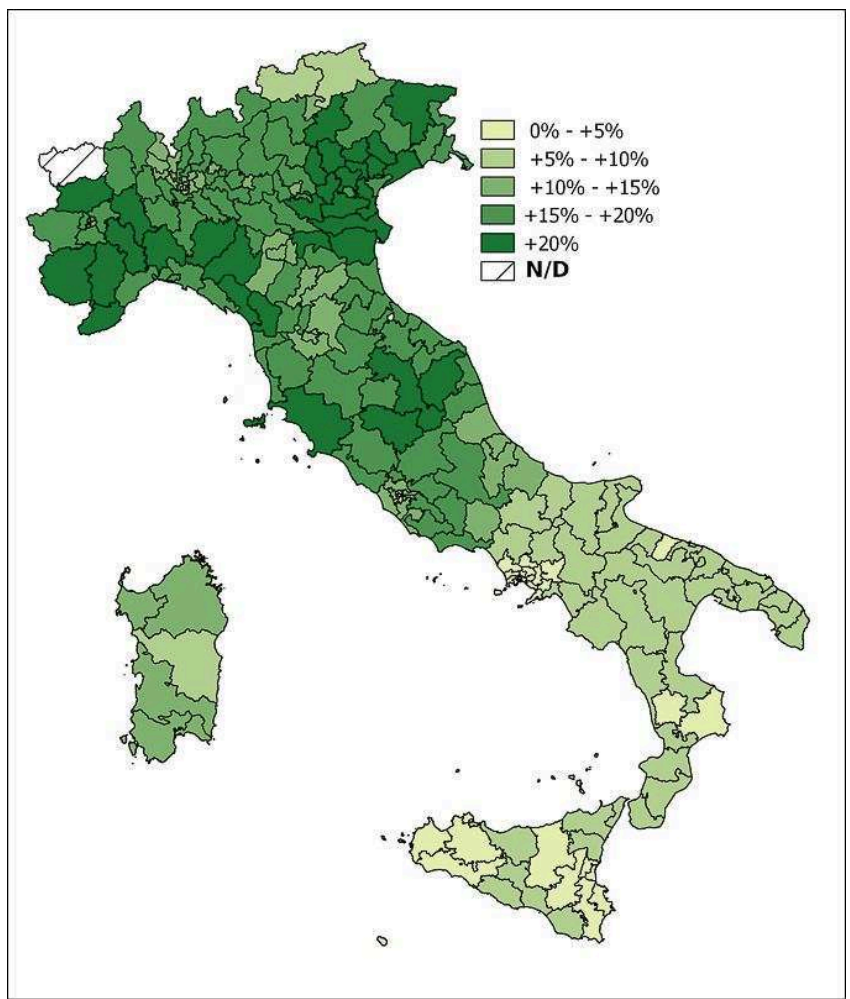

Source : Calculs Quorum/YouTrend sur données du Ministère de l'Intérieur. 
Illustration 12 - Distribution du score du M5S par circonscription (Chambre Basse, élection législative 2018)

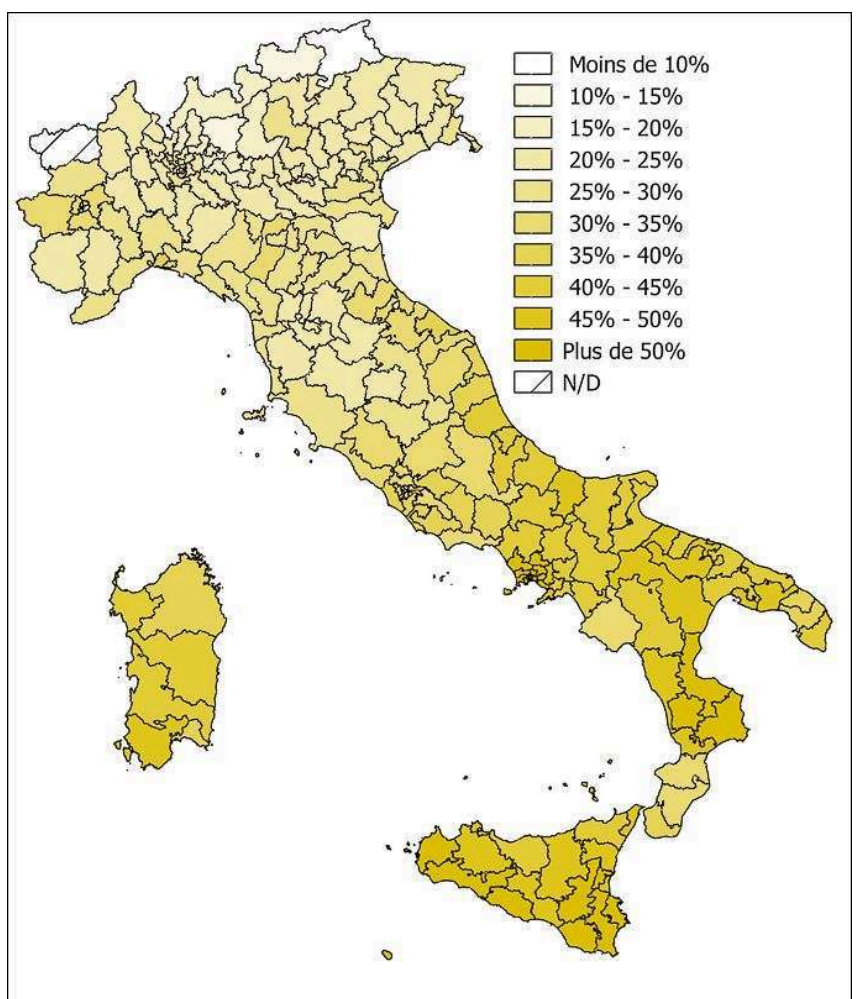

Source : Calculs Quorum/YouTrend sur données du Ministère de l'Intérieur. 
Illustration 13 - Variation du pourcentage de voix obtenu par le M5S par circonscription (Chambre basse, élection législative 2018)

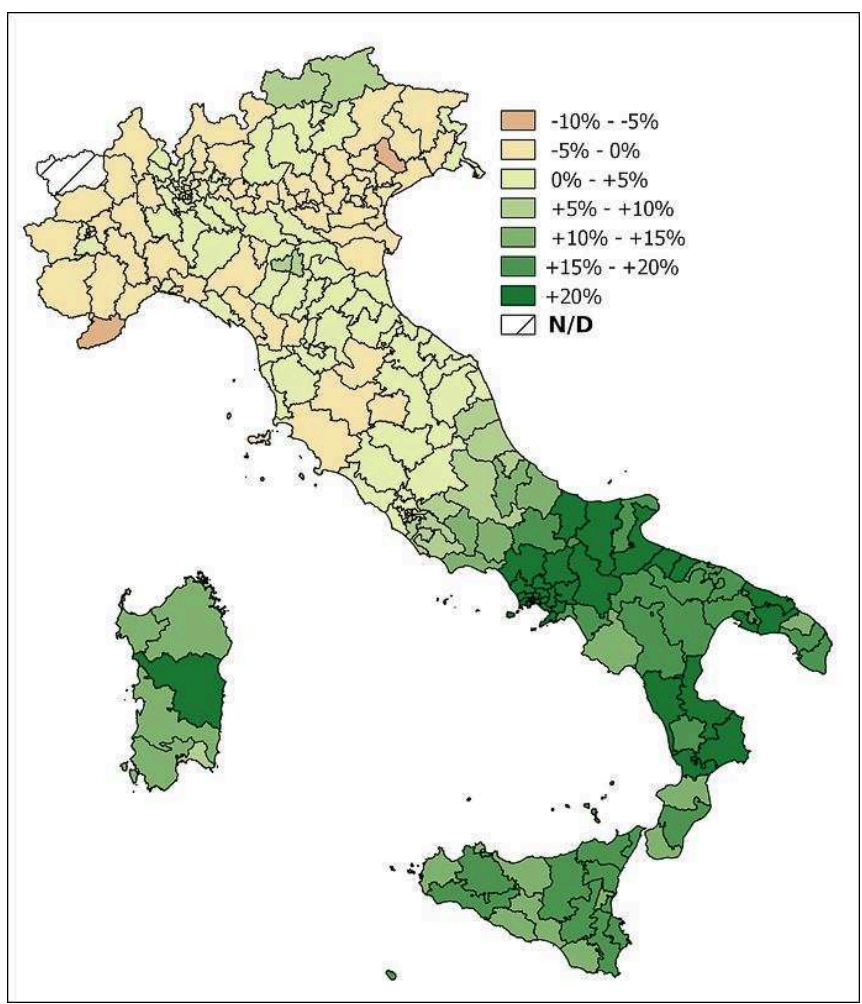

Source : Calculs Quorum/YouTrend sur données du Ministère de l'Intérieur. 
Illustration 14 - Distribution du score du PD par circonscription (Chambre Basse, élection législative 2018)

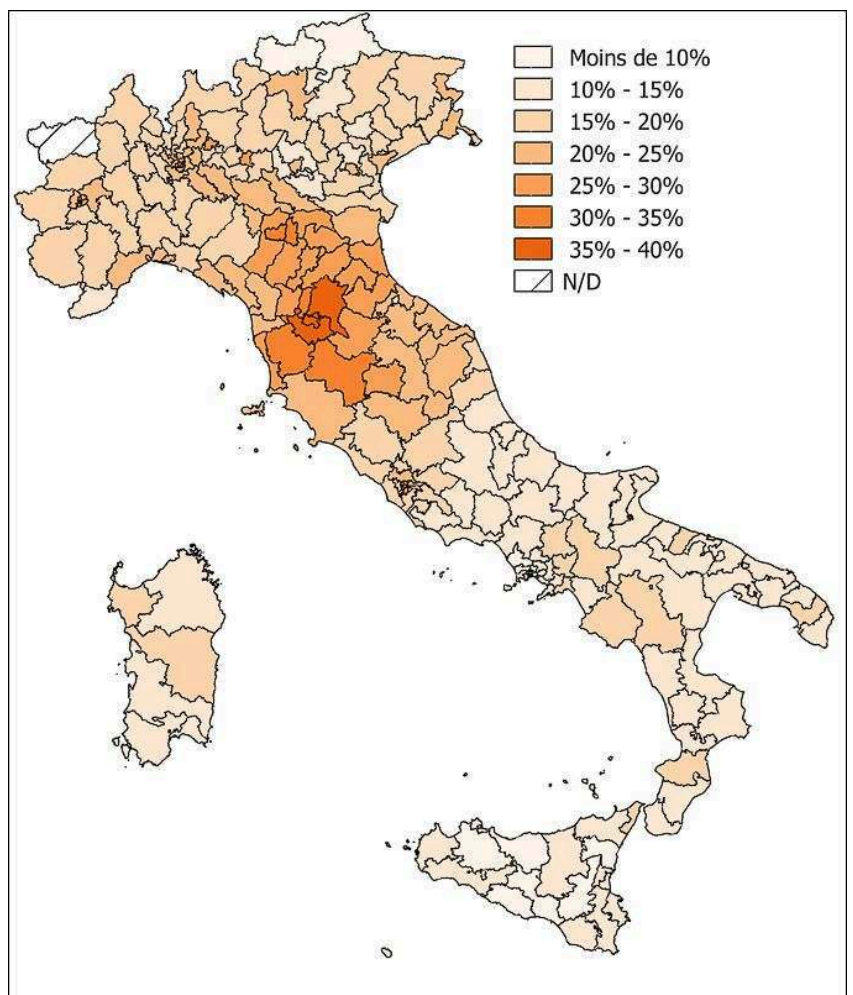

Source : Calculs Quorum/YouTrend sur données du Ministère de l'Intérieur. 
Illustration 15 - Variation du pourcentage de voix obtenu par le PD par circonscription (Chambre basse, élection législative 2018)

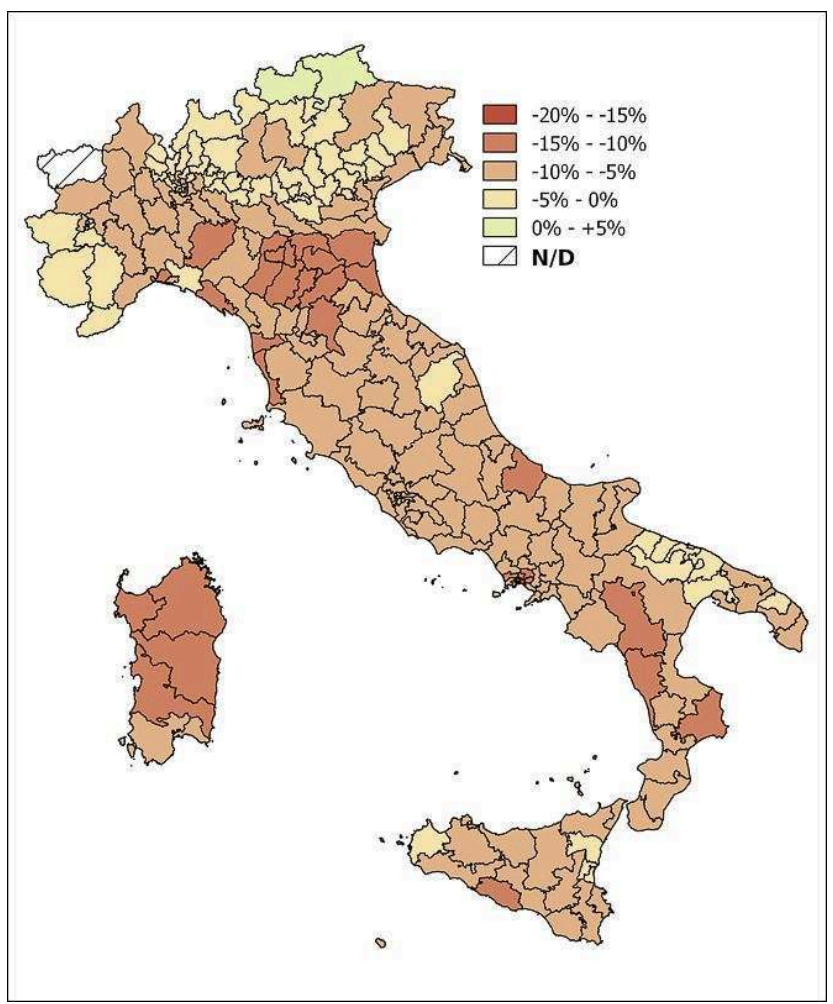

Source : Calculs Quorum/YouTrend sur données du Ministère de l'Intérieur.

Tableau 1 - Résultats des élections législatives du 4 mars 2018 et du 24-25 février 2013

\begin{tabular}{|c|c|c|c|c|c|c|c|c|}
\hline Liste & Traduction & Acronyme & Leader & Coalition & Groupe au PE & Résultat - 2018 & Résultat - 2013 & Différence \\
\hline Lega & Ligue & Ligue & Matteo Salvini & Centre-droit & ENF & $17.4 \%$ & $4.1 \%$ & $13.3 \%$ \\
\hline Forza Italia & Force Italie & FI & Silvio Berlusconi & Centre-droit & PPE & $14.0 \%$ & $21.6 \%$ & $-7.6 \%$ \\
\hline Fratelli d'ttalia & Freres d'talie & Fdl & Giorgia Meloni & Centre-droit & - & $4.4 \%$ & $2.0 \%$ & $2.4 \%$ \\
\hline Noicon l'tialia & Nous avec I'Italie & $\mathrm{NCI}$ & Raffaele Fitto & Centre-droit & PPE & $1.3 \%$ & - & - \\
\hline- & Autres listes de centre-droit & - & - & Centre-droit & & - & $1.6 \%$ & - \\
\hline & TOTAL CENTRE-DROIT & & & & & $37.0 \%$ & $29.2 \%$ & $7.8 \%$ \\
\hline Movimento 5 Stelle & Mouvement 5 Etoiles & M5s & Luigi Di Maio & M5s & EFDD & $32.7 \%$ & $25.6 \%$ & $7.1 \%$ \\
\hline Partito Democratico & Parti Démocrate & PD & Matteo Renzi & Centre-gauche & PSE & $18.8 \%$ & $25.4 \%$ & $-6.7 \%$ \\
\hline Più Europa & Plus Europe & $+E$ & Emma Bonino & Centre-gauche & - & $2.6 \%$ & - & - \\
\hline Insieme & Ensemble & Ens & Giulio Santagata & Centre-gauche & - & $0.6 \%$ & - & - \\
\hline Civica Popolare & Civique Populaire & CIVPOP & Beatrice Lorenzin & Centre-gauche & - & $0.5 \%$ & - & - \\
\hline Südtiroler Volkspartei & Parti populaire sud-tyrolien & SVP & Philipp Achammer & Centre-gauche & - & $0.4 \%$ & $0.4 \%$ & $0.0 \%$ \\
\hline & Autres listes de centre-gauche & - & - & Centre-gauche & & - & $3.7 \%$ & - \\
\hline & TOTAL CENTRE-GAUCHE & & & & & $22.9 \%$ & $29.6 \%$ & $-6.7 \%$ \\
\hline Liberie Uguali & Libres et Egaux & LeU & Pietro Grasso & EXG & PSE/GUE & $3.4 \%$ & - & - \\
\hline- & Autres & - & - & - & - & $4.1 \%$ & $15.7 \%$ & $-11.6 \%$ \\
\hline
\end{tabular}

Élection législative du 4 mars 2018 : Chambre basse - partie proportionnelle. Élection législative du 24-25 février 2013 : Chambre basse.

Source : Ministère de l'Intérieur. 
Tableau 2 - Résultats de l'élection législative du 4 mars 2018 par population de la commune

\begin{tabular}{|c|c|c|c|c|c|c|c|c|c|}
\hline \multicolumn{6}{|c|}{ COALMON } & \multicolumn{4}{|c|}{ LSTES PRINCIPAIES } \\
\hline PCOPULAПLON & CENTRE-DROIT & CENTRE-GAUCHE & MSS & LEU & AUTRES & MES & $P D$ & LECA & $\mathrm{Fi}$ \\
\hline Intergul \& 5.000 hab & $42.3 \%$ & $20.7 \%$ & $29.4 \%$ & $2.6 \%$ & $5.0 \%$ & $29.2 \%$ & $16.9 \%$ & 22.85 & $14.4 \pi$ \\
\hline 5001.15000 & $203 \pi$ & $212 \%$ & $317 \%$ & $29 \%$ & $39 \pi$ & 31. 3\% & $17.8 \%$ & $210 \%$ & $142 \%$ \\
\hline $15.001-25.000$ & $37.6 \%$ & $21.7 \%$ & $33.6 \%$ & 3. $2 \%$ & $3.8 \%$ & $33.2 \%$ & $18.9 \%$ & 18.08 & $14.4 \%$ \\
\hline $25.001-50.000$ & $34.5 \%$ & 21.196 & $36.5 \%$ & 3.48 & 4.180 & $\frac{36.5 \%}{3}$ & $\frac{17.68}{17.08}$ & 14.92 & $15.0 \%$ \\
\hline $50.001 \cdot 100.000$ & 33.78 & $21.4 \%$ & $27.2 \%$ & $30 \%$ & $4.2 \%$ & 37.05 & $17.6 \%$ & 13.28 & $15.3 \%$ \\
\hline $100,001-200,000$ & $33.5 \%$ & $25.3 \%$ & $31.5 \%$ & 4.28 & $4.6 \%$ & 31.25 & $212 \%$ & $15.8 \%$ & 12.05 \\
\hline Plus de 300.000 & $30.32 \%$ & 28.656 & $31.2 \%$ & $50 \%$ & $4.5 \%$ & $30.8 \%$ & $22.5 \%$ & $11.5 \%$ & $13.2 \%$ \\
\hline
\end{tabular}

Source : Calculs Quorum/YouTrend sur données du Ministère de l'Intérieur.

Tableau 3 - Rapport entre les voix obtenues en 2018 et en 2013 par les listes principales selon la population de la commune

\begin{tabular}{|c|c|c|c|c|}
\hline ITALIE & M5S & PD & LEGA & FI \\
\hline Inférieur à $5.000 \mathrm{hab}$ & $163.8 \%$ & $48.4 \%$ & $266.0 \%$ & $86.2 \%$ \\
\hline $5.001-15.000$ & $175.2 \%$ & $51.3 \%$ & $295.5 \%$ & $95.6 \%$ \\
\hline $15.001-25.000$ & $182.9 \%$ & $52.6 \%$ & $344.2 \%$ & $98.5 \%$ \\
\hline $25.001-50.000$ & $190.6 \%$ & $51.5 \%$ & $419.5 \%$ & $99.2 \%$ \\
\hline $50.001-100.000$ & $195.9 \%$ & $51.9 \%$ & $452.1 \%$ & $108.3 \%$ \\
\hline $100.001-300.000$ & $176.0 \%$ & $55.3 \%$ & $390.4 \%$ & $103.3 \%$ \\
\hline Plus de 300.000 & $159.9 \%$ & $59.8 \%$ & $464.5 \%$ & $105.4 \%$ \\
\hline
\end{tabular}

Source : Calculs Quorum / YouTrend sur données du Ministère de l'Intérieur.

Tableau 4 - Matrice de corrélation entre score obtenu par listes et coalitions principales et variables socio-démographiques au niveau de circonscription uninominale (élection législative 2018)

\begin{tabular}{|c|c|c|c|c|c|c|c|c|}
\hline Acronyme & Description & LEGA & $\mathrm{Fl}$ & MES & PD & +EUROPA & $\begin{array}{c}\text { CENTRE- } \\
\text { DROIT }\end{array}$ & $\begin{array}{l}\text { CENTRE- } \\
\text { GAUCHE }\end{array}$ \\
\hline DAB & Densité de population & -0.21 & 0.03 & -0.05 & 0.23 & 0.58 & -0.22 & 0.32 \\
\hline IV & Taux de dépendance des personnes âgées & 0.21 & -0.35 & -0.44 & 0.57 & 0.52 & 0.07 & 0.60 \\
\hline ST & Population étrangere sur 1000 hab. & 0.63 & -0.61 & -0.78 & 0.66 & 0.58 & 0.40 & 0.66 \\
\hline TSO & Taux d'emploi & 0.75 & -0.77 & -0.91 & 0.67 & 0.55 & 0.44 & 0.67 \\
\hline TSOFM & Rapport Taux d'emploi féminin/masculin & 0.55 & -0.71 & -0.82 & 0.74 & 0.69 & 0.26 & 0.76 \\
\hline AAST & Taux d'anal phabetism & -0.67 & 0.62 & 0.76 & -0.47 & -0.58 & -0.42 & -0.49 \\
\hline PDU & Pourcentage de population ayant obtenu un $\mathrm{BAC}+3$ & -0.17 & -0.15 & -0.25 & 0.45 & 0.80 & -0.22 & 0.57 \\
\hline$A D D$ & Nombre d'employés sur 1000 hab. & 0.27 & -0.40 & -0.57 & 0.53 & 0.77 & 0.11 & 0.61 \\
\hline ADDSP & Nombre d'employés publics sur 1000 hab & -0.12 & -0.13 & -0.22 & 0.28 & 0.60 & -0.19 & 0.39 \\
\hline QAI & Pourcentage d'employé dans l'industrie & 0.72 & -0.44 & -0.49 & 0.28 & 0.03 & 0.56 & 0.22 \\
\hline Oss & Pourcentage d'employé dans les services & -0.08 & -0.20 & -0.24 & 0.40 & 0.70 & -0.17 & 0.49 \\
\hline POP NFR & Pourcentage de population née dans une autre région & 0.37 & -0.45 & -0.59 & 0.65 & 0.67 & 0.20 & 0.68 \\
\hline EDI & Pourcentage batiments pre-1945 & 0.07 & -0.12 & -0.28 & 0.34 & 0.36 & -0.01 & 0.38 \\
\hline IVMS & Index de vulnerabilité & -0.75 & 0.55 & 0.77 & -0.44 & -0.38 & -0.58 & -0.45 \\
\hline
\end{tabular}

Source : Calculs Quorum/YouTrend sur données du Ministère de l'Intérieur.

\section{BIBLIOGRAPHIE}

Agenzia Italiana per lo Sviluppo Sostenibile (ASviS), 2018. L'Italia e gli obiettivi di sviluppo sostenibile - Rapporto 2017 [En ligne]. http://asvis.it/public/asvis/files/Rapporto_ASviS_2017/ REPORT_ASviS_2017_WEB.pdf

Albertazzi D., McDonnell D., 2015. Populists in power. London, Routledge, 218 p. 
Alleva G., Celant A., 2018. Struttura urbana e terziario alle imprese. Roma, Sapienza Università Editrice, $190 \mathrm{p}$.

Anderlini F., 2009. Il partito liquido e la durezza del territorio. Il Mulino, Vol. 58, nº 2, p. 199-209.

Bull M. J., Pasquino G., 2018. Italian politics in an era of recession: the end of bipolarism? South European Society and Politics, Vol. 23, $\mathrm{n}^{\circ}$ 1, p. 1-12.

Cataldi M., Emanuele V., 2013. Lo tsunami cambia la geografia e strappa 50 province a Pd e Pdl. In De Sio L., Cataldi C., De Lucia F. (ed.). Le Elezioni Politiche 2013, Dossier Cise, Vol. 4, Rome, CISE, p. 3-55.

Cavallaro M., Diamanti G., Pregliasco L., 2018. Una nuova Italia: Dalla comunicazione ai risultati, un'analisi delle elezioni del 4 marzo. Roma, Castelvecchi Editore.

Cavallaro M., Salza G., Zanetti M., 2018. Il futuro in programma. Analisi quantitativa dei programmi dei partiti alle elezioni politiche 2018. In Cavallaro M., Diamanti G., Pregliasco L., 2018. Una nuova Italia: Dalla comunicazione ai risultati, un'analisi delle elezioni del 4 marzo. Roma, Castelvecchi Editore.

Chiaramonte A., De Sio L. (ed.), 2014. Terremoto elettorale: le elezioni politiche del 2013. Il Mulino, $280 \mathrm{p}$.

Chiaramonte A., Paparo A., 2018. Tra maggioritario e proporzionale : il nuovo sistema elettorale alla prova delle elezioni 2018. Papier présenté au congrès de la Società Italiana di Studi Elettorali, 10-11 mai 2018, Salerno, $30 \mathrm{p}$.

Chiaramonte A., D'Alimonte R., 2018. The new Italian electoral system and its effects on strategic coordination and disproportionality. Italian Political Science, Vol. $13, \mathrm{n}^{\circ} 1$ p. 8-18.

Cerruto M., 2012. La partecipazione elettorale in Italia. Quaderni di Sociologia, Vol. 60, p. 17-39.

Coppola G., Mazzotta F., 2005. I Sistemi Locali del Lavoro: Aspetti teorici ed empirici. CELPE Quaderni di Ricerca, Vol. 2, 82 p.

De Lucia F., Cataldi M., 2013. L'analisi dell'affluenza: una forte accelerazione del declino della partecipazione. In De Sio L., Cataldi C., De Lucia F. (ed). Le Elezioni Politiche 2013, Dossier Cise (4). Rome, CISE, p. 47-52.

De Sio L., 2014. Da dove viene la vittoria di Renzi. In De Sio L., Emanuele V., Maggini N. (ed.). Le Elezioni Europee. Dossier CISE, Vol. 6, Roma, CISE, p. 171-178.

Diamanti G., Ma Pontida è sempre più blu, 2018. YouTrend [En ligne]. http://www.youtrend.it/ 2018/07/04/ma-pontida-e-sempre-piu-blu/

Diamanti I., 2009. Mappe dell'Italia politica: bianco, rosso, verde, azzurro... e tricolore. Il Mulino, Vol. 191, 256 p.

Diamanti I., 2018. La Terza Repubblica che non sa dove andare. La Repubblica [En ligne]. http:// www.repubblica.it/politica/2012/05/09/news/mappe_elezioni_diamanti-34742871/

Duverger M., 1951. The influence of electoral systems on political life. International Social Science Bulletin, vol. 3, n², p. 314-43.

Emanuele V., Vassallo S., 2018. Il voto del 4 marzo tra nuova geografia elettorale ed evoluzione del sistema partitico. Papier présenté au congrès de la Società Italiana di Studi Elettorali, 10-11 mai 2018, Salerno, 25 p.

Galli G., Capecchi V., Cioni Polacchini V., Sivini G., 1968. Il comportamento elettorale in Italia: una indagine ecologica sulle elezioni in Italia fra il 1946 e il 1963. Bologna, Il Mulino, 474 p. 
Gramsci A., 1982. La questione meridionale. Roma, Editori Riuniti, 96 p.

Ignazi P., 1996. The crisis of parties and the rise of new political parties. Party Politics, Vol. 2, $\mathrm{n}^{\circ} 4$, p. 549-566.

Ivaldi G., Lanzone M., Woods D., 2017. Varieties of Populism across a Left-Right Spectrum: The Case of the Front National, the Northern League, Podemos and Five Star Movement. Swiss Political Science Review, Vol. 23, $\mathrm{n}^{\circ}$ 4, p. 354-376.

Lipset S. M., Rokkan S., 1967. Cleavage structures, party systems, and voter alignments: an introduction. In Lipset S. M., Rokkan S. (eds), Party Systems, and Voter Alignments: Cross National Perspective, New York, The Free Press, p. 1-67.

Mannheimer R., Giacomo S., 1987. Il mercato elettorale: identikit dell'elettore italiano. Bologna, Il Mulino, $185 \mathrm{p}$.

Regalia M., 2018. Electoral Reform as an Engine of Party System Change in Italy. South European Society and Politics, Vol. 23, n 1, p. 81-96.

Rolfe M., 2012. Voter turnout: A social theory of political participation. Cambridge, Cambridge University Press, 231 p.

\section{NOTES}

1. Étant donné l'existence de petites différences entre le mécanisme d'élection à la chambre basse et celui du Sénat, nous nous retrouvons avec au moins trois systèmes électoraux différents (Chambre, Sénat, Italiens de l'étranger). Dans cet article, nous nous concentrons sur le fonctionnement du système électoral et les résultats de la Chambre des Députés car cela présente le plus large nombre d'ayant droits, étant donné que l'âge minimal requis pour voter au Sénat est fixé à 25 ans, contre les 18 ans pour le vote dans la chambre baisse.

2. Ces forces, donc, « donnent » les votes à leur coalition, sans bénéficier d'aucun élu. Il y a donc un trade-off entre nombre de listes et sièges obtenus dans la partie proportionnelle : multiplier les listes peut être une stratégie gagnante pour élargir sa propre «offre », mais seulement si ces listes dépassent $1 \%$ des suffrages.

3. Le fait d'utiliser les reformes de la loi électorale de manière stratégique n'est pas un comportement inédit pour les partis politiques italiens, voir Regalia (2018).

4. Le rapport entre le nombre absolu de voix obtenus en 2018 et le nombre absolu de voix obtenus en 2013.

5. Ces changements ont même été «chromatiques »: la couleur de la campagne en 2018 le bleu, sur un style graphique repris de celui de Donald Trump aux Etats-Unis, ce qui correspond à l'abandon du « vert », couleur historique du parti (Diamanti 2018).

6. L'écart moyen entre la moyenne et le résultat de la liste dans chaque circonscription.

\section{RÉSUMÉS}

Cet article décrit et analyse les résultats de l'élection italienne du 4 mars 2018 du point de vue de la géographie électorale. En particulier, nous soulignons l'hétérogénéité de la distribution des 
voix aux principales forces politiques et évoquons l'apparition d'un système politique tripolaire mais imparfait avec deux pôles majeurs (centre-droit et M5S) et un pôle non-compétitif, le centre-gauche. Pour mieux comprendre les caractéristiques territoriales de ces trois pôles, nous observons la relation entre distribution des préférences électorales et distribution des variables sociales et identifions les éléments décrivant ces trois Italies : (i) le pays des petites villes du Nord, à hégémonie liguiste, (ii) l'Italie des grandes villes et des quartiers centraux, où c'est le centre-gauche à gagner, et finalement (iii) les régions pauvres du Sud, des zones de nouvelle installation du M5S.

In this article we describe and analyse the results of the Italian General Election of March 4, 2018. In particular, we focus on the geographical differences in the scores distribution of the main political forces. We argue that, from this point of view, Italy appears as a tripolar-but-imperfect political system with two major poles (centre-right and M5S) and a non-competitive pole, the centre-left. We then observe the relationship between the distribution of electoral preferences and the distribution of a series of socio-demographic variables, in order to identify the main elements describing these "three Italies": (i) the country of the small northern cities, with leghista hegemony, (ii) an Italy made of large and well-educated urban areas, which votes for the centre-left, and finally (iii) the poor regions of the South, newly acquired areas for the M5S.

\section{INDEX}

Mots-clés : Italie, géographie politique, élection, crise politique, système partidaire

Keywords : Italy, political geography, election, political crisis, party system

\section{AUTEURS}

\section{MATTEO CAVALLARO}

Matte Cavallaro, matteo.cavallaro@sciencespo.fr, est partner chez Quorum S.a.S. Il a récemment publié :

- Cavallaro M., Pregliasco L., Vassallo S., 2018. Why the Rosato Law appeared to be proportional and under what conditions it can prove to be majoritarian. Comparative Italian Politics, vol. 10, $\mathrm{n}^{\circ}$ 3, p. 224-242.

- Cavallaro M., Flacher D., Zanetti M., 2018. Radical right parties and European economic integration: Evidence from the analysis of roll-call votes during the 7th term of the European Parliament. European Union Politics, vol. 19, n² 2, p. 321-343.

- Cavallaro M., Diamanti G., Pregliasco L., 2018. Una nuova Italia : dalla comunicazione ai risultati, un'analisi delle elezioni del 4 marzo. Rome, Castelvecchi, 144 p.

\section{DAVIDE POLICASTRO}

Davide Policastro, davide.policastro@agenziaquorum.it, est founding partner chez Quorum S.a.S.

\section{GUIDO SALZA}

Guido Salza, guido.salza@unito.it, est doctorant à l'Université de Milan. Il a récemment publié : - Cavallaro M., Salza G., Zanetti M., 2018. Il futuro in programma. Analisi quantitativa dei programmi dei partiti alle elezioni politiche 2018. In Cavallaro M., Diamanti G., Pregliasco L. Una nuova Italia. Dalla comunicazione ai risultati, un'analisi delle elezioni del 4 marzo. Rome,

Castelvecchi,p. 56-80. 
- Contini D., Salza G., Scagni A., 2017. Dropout and Time to Degree in Italian Universities Around the Economic Crisis. University of Turin, Department of Economics and Statistics, WP 16/17.

\section{MASSIMO ANGELO ZANETTI}

Zanetti, Massimo Angelo, m.zanetti@univda.it, est chercheur à l'Université de la Vallée d'Aoste. Il a récemment publié :

- Cavallaro M., Flacher D., Zanetti M., 2018. Radical right parties and European economic integration: Evidence from the analysis of roll-call votes during the 7th term of the European Parliament. European Union Politics, vol. 19, n 2, p. 321-343.

- Cavallaro M., Salza G., Znetti M., 2018. Il futuro in programma. Analisi quantitativa dei programmi dei partiti alle elezioni politiche 2018. In Cavallaro M., Diamanti G., Pregliasco L., Una nuova Italia. Dalla comunicazione ai risultati, un'analisi delle elezioni del 4 marzo. Rome, Castelvecchi, p. 56-80.

- Zanetti M., 2018. Lavoro flessibile e percorsi lavorativi. Un'indagine esplorativa. Turin, Accademia University Press, p. 1-281. 